\title{
Capítulo 7 Análisis paramétrico de los elementos del actuador chevrón
}

\section{Chapter 7 Parametric analysis of the Chevron actuator}

TECPOYOTL-TORRES, Margarita $\dagger^{*}$, RODRIGUEZ-FUENTES, Sahiril Fernanda, KOSHEVAYA, Svetlana y VARGAS-CHABLE, Pedro

Centro de Investigación en Ingeniería y Ciencias Aplicadas, Universidad Autónoma del Estado de Morelos (UAEM)

Licenciatura en Tecnología con áreas terminales en Física Aplicada y Electrónica, Instituto de Investigación en Ciencias Básicas y Aplicadas, UAEM

Facultad de Ciencias Químicas e Ingeniería, UAEM

ID $1^{\mathrm{er}}$ Autor: Margarita, Tecpoyotl-Torres/ ORC ID: 0000-0002-4336-3771, CVU CONACYT ID: 20650.

ID $1^{\text {er }}$ Coautor: Sahiril, Rodríguez-Fuentes/ ORC ID: 0000-0002-1587-3053

ID $2^{\text {do }}$ Coautor: Svetlana, Koshevaya / ORC ID: 0000-0002-5966-1343, CVU CONACYT ID: 15967

ID $3^{\text {er }}$ Coautor: Pedro, Vargas-Chable/ ORC ID: 0000-0002-2290-3740, CVU CONACYT ID: 484392

DOI: $10.35429 /$ H.2020.5.117.140

M. Tecpoyotl, S. Rodriguez, S. Koshvaya y P. Vargas

tecpoyotl@uaem.mx

A. Marroquín, J. Olivares, L. Cruz y A. Bautista. (Coord) Ingeniería. Handbooks-@ECORFAN-Mexico, Querétaro, 2020. 


\section{Resumen}

Los sistemas microelectromecánicos (MEMS) se forman de componentes eléctricos y mecánicos. Se fabrican utilizando tecnologías de micromaquinado, con base en la tecnología de circuitos integrados. Los MEMS son ampliamente utilizados en la vida diaria, en particular se destaca su uso en sectores como, el automotriz, médico, de rehabilitación, etc. Entre los dispositivos que se desarrollan, se detectan constantemente sensores y actuadores nuevos o mejorados. Además, hay muchos grupos de investigación interesados en el desarrollo de los MEMS. Actualmente, para la optimización de los diseños, es posible desarrollar análisis paramétricos, mediante los cuales, se realizan barridos automatizados de forma rápida, lo cual genera información útil para analizar las tendencias de desempeño y, por tanto, para seleccionar los valores más adecuados de las variables, de acuerdo con los requisitos establecidos. La parametrización reduce tanto el tiempo, como los costos involucrados en el proceso de diseño. En este capítulo, analizamos la parametrización de los elementos básicos de un actuador chevrón, así como sus efectos sobre el desplazamiento y la fuerza de reacción en la flecha, para cada caso. Con base en los resultados obtenidos, usando ANSYS, se determina la tendencia de desempeño de los elementos bajo análisis y se muestra de manera gráfica.

\section{Desplazamiento; fuerza; parametrización; ANSYS; número de brazos.}

\section{Introducción}

A partir de 1960, se experimentó con el uso de tecnologías de fabricación de Circuitos Integrados (CIs), para desarrollar microestructuras mecánicas. Entre los primeros dispositivos, se encuentra el transistor de compuerta resonante (RSG, por sus siglas en inglés), producido por Nathenson en Westinghouse. Este transistor combina las ventajas de contar con un factor de alta calidad mecánica y la ganancia intrínseca de un transistor (Tazabekov, 2015), fue el primer dispositivo microelectromecánico (MEM) fabricado, pero no fue comercializado exitosamente. Fue hasta la década de los 70`s que se logró la adopción comercial de sensores de presión y acelerómetros (Kaajakari, 2009).

A la clase de micro dispositivos o sistemas integrados que, relacionan componentes eléctricos y mecánicos, desarrollados mediante tecnologías de micromaquinado, basadas en la tecnología de los CIs, son llamados Sistemas microelectromecánicos (MEMS). Su tamaño varía desde micrómetros hasta milímetros. Actualmente, se desarrollan muchos procesos únicos de micromecanizado específicos para MEMS. Estas nuevas tecnologías, junto con el diseño de nuevos o mejorados dispositivos, han permitido un acelerado desarrollo de mercados para los MEMS, donde cada año, ingresan nuevos productos cuyos costos son, en su mayoría, accesibles.

La comercialización de dispositivos MEMS producidos para la industria automotriz, se inició entre 1970 y 1980, por varias compañías (por ejemplo, transductores IC, Foxboro ICT, Dispositivos transitorios, sensores IC y Novasensor) (Judy, 2001). En los últimos años, los MEMS se han convertido en un componente vital dentro de una amplia gama de tecnologías, lo que hace que el estudio de su rendimiento y fiabilidad operativa sea un aspecto crítico para su correcta funcionalidad.

Actualmente, se fabrican una amplia variedad de dispositivos MEMS, que se aplican a varios sistemas que se utilizan en la vida diaria, por ejemplo, en automóviles, periféricos de computadoras, impresoras de inyección de tinta, cámaras fotográficas, videojuegos, etc. También se aplican en monitoreo ambiental, aplicaciones biológicas, tratamientos médicos, etc. (Cauchi, y otros, 2019). De acuerdo con el mercado que atienden, se destaca su uso en la industria automotriz, electrónica de consumo, defensa militar y aeronáutica, medicina, el sector industrial en general y las telecomunicaciones (Yole Developpement, 2019).

Los MEMS pueden actuar como sensores o actuadores, ya sea recibiendo información del medio, o como actuadores, transformando algún tipo de energía en una acción determinada. En un actuador, en general, una acción mecánica con una fuerza y movimiento (traslacional o rotacional) determinados, se activa en respuesta a una señal de control.

Hay actuadores que existen en la naturaleza, en particular, en músculos de animales o en plantas. La naturaleza ha sido la fuente de la llamada bio-inspiración, con base en la cual, diversos sistemas, tales como amplificadores de desplazamiento o algunos elementos robóticos, han sido diseñados. 
Entre los actuadores hechos por el hombre, se encuentran los hidráulicos, neumáticos y solenoides. Otros, se basan en materiales que cambian su forma, tales como piezoeléctricos, de memoria de forma y magnetoresistivos (Huber, Fleck, \& Ashby, 1997). Algunos actuadores operan de forma lineal, causando un cambio finito en la longitud, mientras que otros, como los motores, pueden producir desplazamientos considerados infinitos.

Los actuadores basados en MEMS tienen una amplia gama de aplicaciones, tales como microinterruptores, microespejos y micromotores. Los esquemas de actuación más utilizados son actuación térmica, electrostática, magnética, piezoeléctrica y neumática. Se destaca la actuación térmica debido a que genera un alto nivel de desplazamiento, alta precisión, repetibilidad y simplicidad en el diseño. Esta actuación puede obtenerse de varias formas (Elbuken, Gui, Ren, Yavuz, \& Khamesee, 2008), entre las más comunes, se encuentra la actuación electrotérmica, mediante la cual, se han desarrollado diversos dispositivos, entre ellos, micropinzas (Wang, Shen., \& Chen, 2015).

La actuación electrotérmica consiste en la expansión térmica de los brazos de sujeción, debido al Efecto de Joule, en presencia de corriente eléctrica. Las micropinzas electrotérmicas tienen muchas ventajas, tales como una amplia deformación, intensa fuerza de sujeción, bajo voltaje de operación y estructura compacta. Las limitaciones de los actuadores electrotérmicos están relacionadas con la alta temperatura en la región cercana a los objetivos de sujeción, por lo que deben usarse brazos de sujeción largos que disipen el calor producido por los actuadores (Iamoni \& Somà, 2014), entre otras estrategias que han sido empleadas. En la literatura pueden hallarse varios ejemplos de micropinzas actuadas mediante actuadores chevrón.

\subsection{El actuador chevrón}

Los actuadores de tipo Chevrón, también llamados actuadores térmicos con brazos- $\mathrm{V}$, o actuadores de viga flexionada, se encuentran entre los actuadores MEMS más conocidos. Un actuador chevrón consiste básicamente de un arreglo de pares de brazos, anclados en sus extremos exteriores, con un ángulo inicial predefinido $(\theta)$, y una flecha, también llamada haz central, masa de prueba o vástago movible, la cual permite el acoplamiento de los brazos. El actuador se calienta por el Efecto de Joule, debido al paso de corriente a través de la estructura, generada al aplicar una diferencia de potencial en los contactos anclados al substrato (anclas). Cuando los brazos se calientan, se expanden y tienden a deformarse para generar un movimiento lineal uniforme en la flecha. El ángulo predefinido de los brazos direcciona al movimiento de la flecha. En la Figura 7.1 se muestra un actuador chevrón de 2 brazos. Al ángulo predefinido, también se le conoce como ángulo inicial, de inclinación o de apertura.

Figura 7.1 Elementos de un actuador chevrón de dos brazos



Fuente de Consulta: Elaboración propia

Los actuadores chevrón fueron implementados por primera vez por L. Que in 1999 y M.J. Sinclair en 2000 (Baracu, y otros, 2015). L. Que in 1999, describió a microactuadores chevrón, con respuesta en el plano (paralelo al substrato), con fuerzas de alrededor de un mili-Newton, mientras que M. J. Sinclair en 2000, propuso un actuador chevrón para la medición de la temperatura.

El actuador chevrón se ha utilizado para la medición in situ de la resistencia a la fractura de películas delgadas MEMS (Zhuo, Yueqing, Xinyu, \& Xuping, 2015). Otro de sus usos comunes es su integración, de manera individual, o como arreglos de chevrones en micropinzas usadas ampliamente en tareas de microensamblaje y micromanipulación. 
Las micropinzas actúan como elementos clave para manejar objetos frágiles, como células vivas, nanoalambres, piezas micromecánicas, entre otros objetivos de manipulación (Yukun, Nimping, \& Qingsong, 2014). También se han desarrollado microespejos con base en arreglos de actuadores chevrón. (Baracu, y otros, 2015)

\subsection{Diseño paramétrico}

En el diseño de un dispositivo, es necesario identificar a los parámetros fundamentales de diseño, así como identificar a los efectos de sus variaciones en su desempeño, para finalmente, tomar las decisiones adecuadas, con base en la información obtenida a partir de las herramientas adecuadas (ANSYS Inc., 2011).

Para el conocimiento de las variaciones de los parámetros de diseño, puede realizarse el análisis de manera manual, o bien, mediante simulación, de manera puntual o discreta, pero deben hacerse varias simulaciones para obtener información de un amplio rango de variación, caso por caso, lo que lleva mucho tiempo y esfuerzo adicional. Afortunadamente, herramientas como ANSYS, entre otras, hacen posible que se realice el análisis de las variaciones de interés, de una manera relativamente sencilla, mediante barridos paramétricos automatizados de las variables bajo análisis, con iteraciones rápidas, mediante la aplicación del Método de Elemento Finito. Es decir, el software de simulación, en el análisis paramétrico resuelve de manera automática rangos completos de variables específicas. El desplegado gráfico de resultados permite al usuario, detectar las tendencias, al analizar el desempeño mostrado, y elegir los valores de las variables que sean más apropiadas, de acuerdo con los requerimientos establecidos, con base en las necesidades de la aplicación a la que serán destinados.

El diseño paramétrico reduce considerablemente el tiempo y costo de desarrollo de los procesos de diseño, a la vez que guía el desarrollo de un diseño robusto. En los procesos de diseño de sistemas complejos, es común seguir metodologías de diseño probadas, tales como el Modelo V o Smart product system design model, donde la etapa intermedia, que recibe el nombre de Detailed Design \& Optimization (ANSYS Inc., s.f.), es la directamente relacionada con el análisis paramétrico.

En este capítulo, los actuadores son implementados en Silicio, con los cuales se analizará paramétricamente a sus elementos geométricos, con la finalidad de contar con una amplia base de información que apoye en el diseño de un actuador chevrón.

La hipótesis central es la siguiente:

Al llevar a cabo la parametrización de los elementos del actuador chevrón, puede conocerse la tendencia de desempeño del actuador, con base en el desplazamiento y la fuerza de reacción de la flecha, permitiendo la selección, por parte del diseñador, de las dimensiones más adecuadas de los elementos bajo análisis, teniendo en cuenta, además, a los requerimientos establecidos por la aplicación, así como por el proceso de fabricación.

Este trabajo se divide en las siguientes secciones:

Sección 1. Se plantea brevemente al desarrollo histórico de los MEMS y de su uso en la vida cotidiana, tanto de sensores, como de actuadores. Se describe también, de manera particular, al surgimiento de los actuadores chevrón, así como algunas de sus principales aplicaciones.

Sección 2. En esta sección se establecen, las principales características del dispositivo, las ecuaciones que rigen su comportamiento, así como los parámetros físicos y mecánicos del material utilizado para su implementación.

Sección 3. Se lleva a cabo el análisis de los resultados, tanto teóricos, como los obtenidos mediante simulación. Los parámetros de interés son: el número de brazos, el ángulo de inclinación, el ancho y largo de los brazos y el grosor de la estructura. Se analiza también al efecto del gradiente de temperatura sobre algunos elementos específicos. La respuesta se analiza, básicamente, con relación al desplazamiento y a la fuerza de reacción. 
Sección 4. Se presenta un anexo en el que se describe un ejemplo del proceso de parametrización realizado en ANSYS.

Sección 5. Se presentan las conclusiones, destacando a los resultados sobresalientes, así como algunas observaciones finales y se plantea trabajo futuro.

Sección 6. Se agradece el apoyo recibido para llevar a cabo a este trabajo.

Sección 7. Se muestran las referencias bibliográficas consultadas.

\subsection{Modelado físico del dispositivo}

En este capítulo, se utiliza Silicio como material estructural para la implementación de los actuadores chevrón. Las propiedades físicas y mecánicas de este material se muestran en la Tabla 7.1.

Tabla 7.1 Parámetros físicos y mecánicos del Silicio

\begin{tabular}{|l|l|r|}
\hline Parámetro [unidad] & Descripción & Valor \\
\hline $\boldsymbol{\rho}\left[\mathbf{k g} / \mathbf{m}^{\mathbf{3}}\right]$ & Densidad & 2329 \\
\hline $\mathbf{E}[\mathbf{G P a}]$ & Módulo de Young & 130.1 \\
\hline $\boldsymbol{\alpha}[\mathbf{1} / \mathbf{K}]$ & Coeficiente de expansión térmica & 2.568 e-6 \\
\hline $\boldsymbol{\kappa}[\mathbf{W} / \mathbf{m} * \mathbf{K}]$ & Conductividad térmica & 148 \\
\hline $\boldsymbol{v}[\mathbf{a d i m e n s i o n a l}]$ & Razón de Poisson & 0.33 \\
\hline $\mathbf{C} \mathbf{p}\left[\mathbf{J} / \mathbf{~ k g}^{*} \mathbf{K}\right]$ & Calor específico & 712 \\
\hline $\boldsymbol{\rho}\left[\mathbf{\Omega}^{*} \mathbf{m}\right]$ & Resistividad & 1.5 e-4 \\
\hline Punto de fusion, $\left[{ }^{\circ} \mathbf{C}\right]$ & Punto de fusión & $1414 \mathrm{C}$ \\
\hline Esfuerzo último, $[\mathbf{M P a}]$ & Esfuerzo último & 250 \\
\hline
\end{tabular}

Fuentes de Consulta: (Vargas-Chable, Tecpoyotl-Torres, Robles-Casolco, \& Cabello-Ruiz, 2015), (Yang \& Qingsong, 2017), (Jui-Chang, Hen-Wei, Shu-Wei, \& Yao-Joe, 2014)

Es importante mencionar que el Silicio es uno de los materiales más comunes para la producción de dispositivos MEMS. Entre sus principales caracetrísticas se encuentran su alto punto de fusión $\left(1414^{\circ} \mathrm{C}\right)$, su baja expansividad, así como la alta pureza con la que puede obtenerse. Debe señalarse, que su coeficiente de expansión térmica no es constante. (Swenson, 1983) propone una relación suave, obteniendo un comportamiento creciente del coeficiente de expansión térmica, conforme se incrementa la temperatura de $90 \mathrm{~K}$ a $890 \mathrm{~K}$. Además, proporciona una tabla con los valores discretos del coeficiente de temperatura, en el rango de temperatura considerado.

Para aplicaciones a nivel macrométrico, también pueden hallarse los valores de sus parámetros mecánicos de otros materiales, en la literatura. Por ejemplo, en (Araujo, Briceño, \& Rondón, 2020), se presenta el caso de mezclas asfálticas. Mientras que, en (González, Martínez, \& Graciano, 2020), se analiza el caso de resinas epóxicas y Kevlar.

El actuador chevrón básico está conformado por dos brazos, sus dimensiones se muestran en la Tabla 7.2. Su simulación se lleva a cabo en el software ANSYS Workbench, para obtener el desplazamiento y la fuerza resultante, parametrizando a los siguientes elementos del actuador:

- Ángulo de inclinación

- Largo y ancho del brazo

- Largo y ancho de la flecha

- Grosor de la estructura

Se analiza, además, al efecto de la parametrización del gradiente de temperatura sobre el desplazamiento y la fuerza de reacción de la flecha. 
Debe señalarse que la selección de elementos a parametrizar se realiza a partir de aquellos que influyen significativamente en las variables de interés, del dispositivo bajo análisis. En el caso de (Araujo, Briceño, \& Rondón, 2020), debido a que se enfocan en probetas fabricadas con mezclas asfálticas, la parametrización se realiza sobre los diámetros, espesores y condiciones de apoyo. Para el caso de los elementos a parametrizar en un tren de aterrizaje, pueden consultarse en (González, Martínez, \& Graciano, 2020). Mientras que, sobre el comportamiento a fatiga de la aleación UNS A97075-T6 (ALZn), en (Béjar, 2019), se lleva a cabo el análisis, con enfoque en la predicción del número de ciclos; desarrollando los modelos paramétricos correspondientes.

En nuestro caso, la cantidad de movimiento de la flecha de un actuador chevrón depende del ángulo de inclinación $\theta$, de la geometría y del número de brazos $(N)$, así como del Módulo de Young $E$. Para el cálculo de su desplazamiento, de acuerdo con el número de brazos $N$, se utiliza (Zhu, Corigliano, \& Espino, 2016):

$U_{y}=\frac{F_{y}}{N\left(S^{2} \frac{E A}{L}+C^{2} \frac{12 E I}{L^{3}}\right)}$

donde $F_{y}$ es la fuerza de actuación [N], $S$ es la función seno del ángulo de apertura de cada brazo, $C$ es el coseno del ángulo de apertura de cada brazo, $I$ es el Momento de inercia $\left[\mathrm{m}^{4}\right], A$ es el área de sección de transversal del brazo $\left[\mathrm{m}^{2}\right]$ y $L$ es la longitud del brazo $[\mathrm{m}]$.

Tabla 7.2 Parámetros de diseño del actuador chevr

\begin{tabular}{|c|c|c|}
\hline Variable & Descripción & $\begin{array}{l}\text { Tamaño } \\
{[\mu \mathrm{m}]}\end{array}$ \\
\hline$L_{b}$ & Largo del brazo & 600 \\
\hline$w_{b}$ & Ancho del brazo & 5 \\
\hline$t_{b}$ & Grosor de toda la estructura & 15 \\
\hline$w_{f}$ & Ancho de la flecha & 30 \\
\hline$L_{f}$ & Largo de la flecha & 65 \\
\hline $\boldsymbol{\theta}$ & Ángulo de inclinación & 0.9 \\
\hline
\end{tabular}

Fuente de consulta: (Vargas Chable, Ferrara Bello, Tecpoyotl Torres, Varona, \& Sandoval Reyes, 2019)

Para calcular al desplazamiento, considerando a un gradiente de temperatura $[\Delta T]$, se utiliza a la ecuación 2 (Yong, Corigliano, \& D Espinosa, 2006):

$U^{\Delta T}=\alpha \Delta T L \frac{s}{\left(s^{2}+c^{2} \frac{12 I}{A L^{2}}\right)}$

donde $\alpha$ es el coeficiente de expansión térmica $[1 / \mathrm{K}], \Delta T$ es el gradiente de temperatura $\left[{ }^{\circ} \mathrm{K}\right]$ y $L$ es la longitud del brazo [m].

El momento de inercia, para el caso de un haz o brazo de ancho $w$ y grosor $t$, se calcula de acuerdo con la ecuación 3, (Kaajakari, 2009):

$I=\frac{w^{3} t}{12}$

Para obtener la fuerza, se utiliza la ecuación 4, o bien, directamente la Ley de Hooke:

$\boldsymbol{F}_{\boldsymbol{y}}=N \alpha E A \Delta T \sin \theta$

donde el ángulo $\theta$ corresponde al ángulo de inclinación del brazo $\left[{ }^{\circ}\right]$ y $\alpha$ al coeficiente de expansión térmica.

La resistencia de la viga se da por: 
$R=\frac{\rho L}{A}$

Donde $\rho$ es la resistividad. $L$ y $A$ fueron previamente definidas (Báez Álvarez, 2011).

El cálculo de la constante de rigidez puede realizarse mediante la ecuación 6, (Trung Hoang, Tien Nguyen, \& Hong, 2019):

$k=\frac{2 N E\left(12 I \cos ^{2} \theta+A L^{2} \sin ^{2} \theta\right)}{L^{3}}$

\subsection{Resultados}

Con el actuador chevrón de 2 brazos, se lleva a cabo la parametrización de sus elementos. Se eligió un grosor de $15 \mu \mathrm{m}$, ya que es común en la literatura. Además, se considera una temperatura máxima de $78^{\circ} \mathrm{C}$, evitando el uso de temperaturas que pudiesen afectar a los objetivos con los que se tenga interacción. Se obtiene así un cambio de temperatura de $56{ }^{\circ} \mathrm{C}\left(\Delta \mathrm{T}=78-22{ }^{\circ} \mathrm{C}\right)$, donde $22{ }^{\circ} \mathrm{C}$ es la temperatura ambiente que da por default ANSYS. Los valores de los elementos del actuador se toman de la Tabla 7.2, y se realiza la parametrización sobre el elemento bajo análisis, en cada caso.

\subsection{Parametrización de la longitud de brazo}

Se realizó un barrido de 100 a $1000 \mu \mathrm{m}$. El gráfico 7.1, muestra el desplazamiento de la flecha, correspondiente a las longitudes de brazo consideradas en el barrido. Puede apreciarse una alta linealidad en el comportamiento a partir de $250 \mu \mathrm{m}$. El error entre los resultados teóricos y la simulación es aceptable para todos los casos, siendo 9.1\%, el porcentaje de error más alto, que corresponde a $1000 \mu \mathrm{m}$. Este bajo error, permite validar, mediante la herramienta ANSYS, el modelo analítico utilizado. Cabe señalar que, comúnmente en la bibliografía se utilizan longitudes de brazo en un rango de 200 a $400 \mu \mathrm{m}$. La selección de la longitud se realiza generalmente, de acuerdo con las necesidades de desplazamiento que se requiera en la aplicación específica.

Gráfico 7.1 Longitud de brazo contra desplazamiento



Fuente de Consulta: Elaboración propia

Con relación al efecto de la longitud de brazo sobre la fuerza, no se observa un cambio significativo, ya que de acuerdo con la ecuación 4, se considera constante al Módulo de Young. En cálculos donde se incluya un gradiente de temperatura, podría observarse un ligero cambio.

\subsection{Parametrización del ancho del brazo}

En el gráfico 7.2, se presenta la relación inversamente proporcional entre el ancho del brazo y el desplazamiento de la flecha, de acuerdo con (Kwan, y otros, 2012). 
Puede observarse que, si se incrementa el ancho del brazo disminuye el error entre la aproximación analítica y los resultados obtenidos mediante simulación. El error máximo corresponde a 8.3\% para el ancho mínimo bajo análisis. Se sugiere utilizar anchos mayores a $5 \mu \mathrm{m}$, y de hasta $10 \mu \mathrm{m}$, esto debido a que más allá de este valor podría resultar impráctico, puesto que incrementaría considerablemente a la longitud de la flecha, lo que, a su vez, llevaría a aumentar la resistencia de la viga, de acuerdo con la ecuación (5). Se observa que, para todos los valores de anchura considerados existe un comportamiento decreciente.

Con relación a la variación del ancho del brazo con la fuerza que se genera en la punta de la flecha, el error entre la aproximación analítica y los resultados de la simulación, se mantiene dentro de un rango aceptable (menor al 9\%). Se sugiere nuevamente que, el ancho del brazo se encuentre en un rango de 3 a $10 \mu \mathrm{m}$, con la finalidad de que las dimensiones sean prácticas, evitando áreas excesivamente grandes. Se muestra un comportamiento con una alta linealidad en todo el rango bajo análisis. Nuevamente, la aplicación para la que se requiere será determinante en la selección.

Gráfico 7.2 Ancho de brazo contra desplazamiento

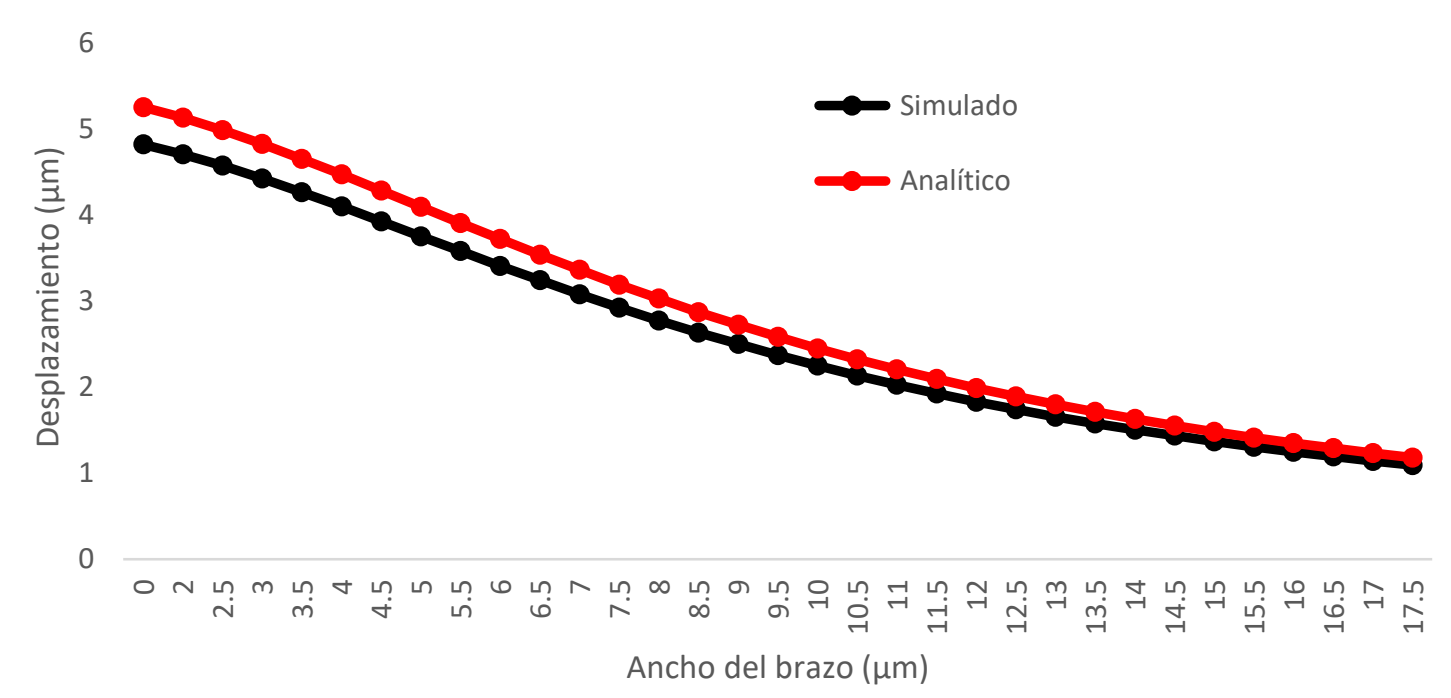

Fuente de Consulta: Elaboración propia

Gráfico 7.3 Ancho de brazo contra fuerza

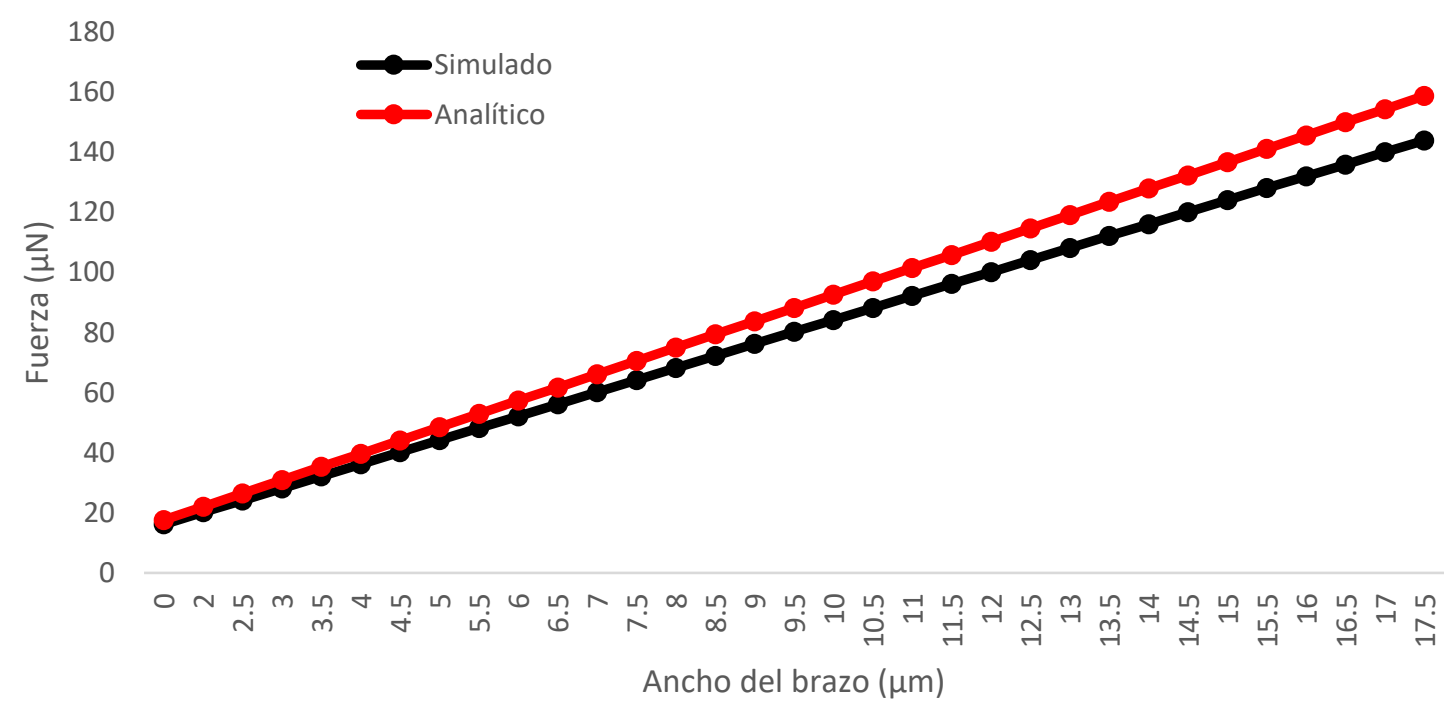

Fuente de Consulta: Elaboración propia 


\subsection{Parametrización del ángulo de inclinación del brazo}

En el gráfico 7.4, se muestra la relación entre ángulo de inclinación y el desplazamiento. Puede observarse que, el porcentaje más alto de error entre los valores corresponde a $8.7 \%$ (entre $0.4^{\circ}$ y $0.5^{\circ}$ ). Las curvas muestran un comportamiento no lineal, siendo creciente hasta $0.5^{\circ}$ y decreciente más allá de este valor. Para ángulos en el rango de $0.1^{\circ}$ hasta $0.3^{\circ}$, se observa un alto incremento en el desplazamiento, sin embargo, la selección de ángulos muy reducidos puede enfrentar problemas de resolución en los procesos de fabricación (Potekhina \& Wang, 2019).

Gráfico 7.4 Ángulo de inclinación contra desplazamiento



Fuente de Consulta: Elaboración propia

Con relación a la variación entre el ángulo de inclinación y la fuerza, mostrada en el gráfico 3.5, se observa nuevamente un comportamiento lineal. El error en $0.1^{\circ}$ es $5.3 \%$, mientras que en $0.9^{\circ}$ es de $8.8 \%$. Para la selección del ángulo en este rango, deben considerarse nuevamente a las limitaciones del proceso de fabricación y la aplicación a la que se destine.

Gráfico 7.5 Ángulo de inclinación contra fuerza



Fuente de consulta: Elaboración propia

Debido a que en la literatura se hallaron valores mayores a los ángulos analizados anteriormente, se realiza una nueva parametrización para valores de ángulo de $1^{\circ}$ a $10^{\circ}$. En (Báez Álvarez, Linares Aranda, Calleja Arriaga, \& Molina Reyes, 2013), se proponen 3 diseños de actuador chevrón, en los que considera un ángulo de $6^{\circ}$ en uno de ellos, mientras que en los 2 restantes de $10^{\circ}$. El gráfico 3.6 muestra la relación entre ángulo y desplazamiento, en la que se observa, un comportamiento decreciente conforme se incrementa el tamaño del ángulo. 
El mayor error es del 8.4\%. La sugerencia es utilizar ángulos menores a $3^{\circ}$ debido al amplio desplazamiento obtenido en la flecha, sin embargo, de acuerdo con la aplicación, debe considerarse también a la fuerza proporcionada (vea Gráfico 7.7).

Gráfico 7.6 Ángulo de inclinación contra desplazamiento

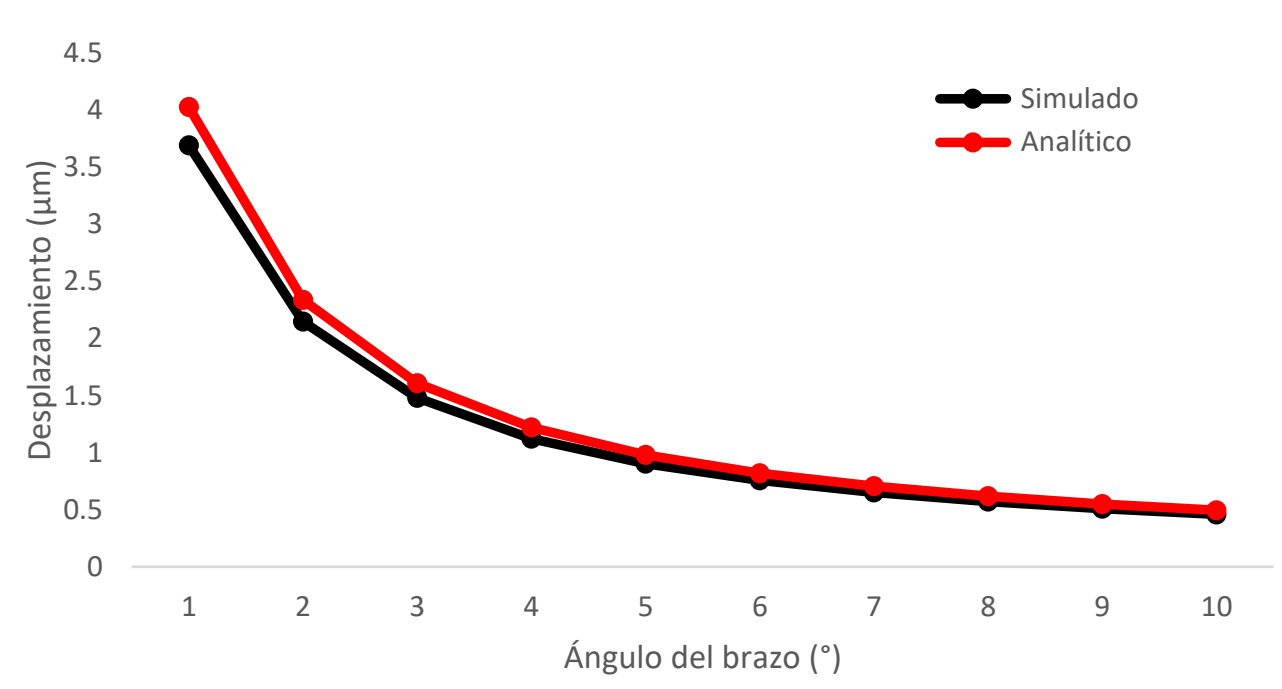

Fuente de Consulta: Elaboración propia

En el gráfico 7.7 se observa a la relación del ángulo con la fuerza, se observa que se trata de una relación lineal y que, si lo que se requiere es fuerza, se sugiere utilizar ángulos mayores, de acuerdo con la necesidad a atender. Cabe señalar que, el error máximo se encuentra en $10^{\circ}$ y corresponde a $9.6 \%$.

Gráfico 7.7 Ángulo de inclinación contra fuerza

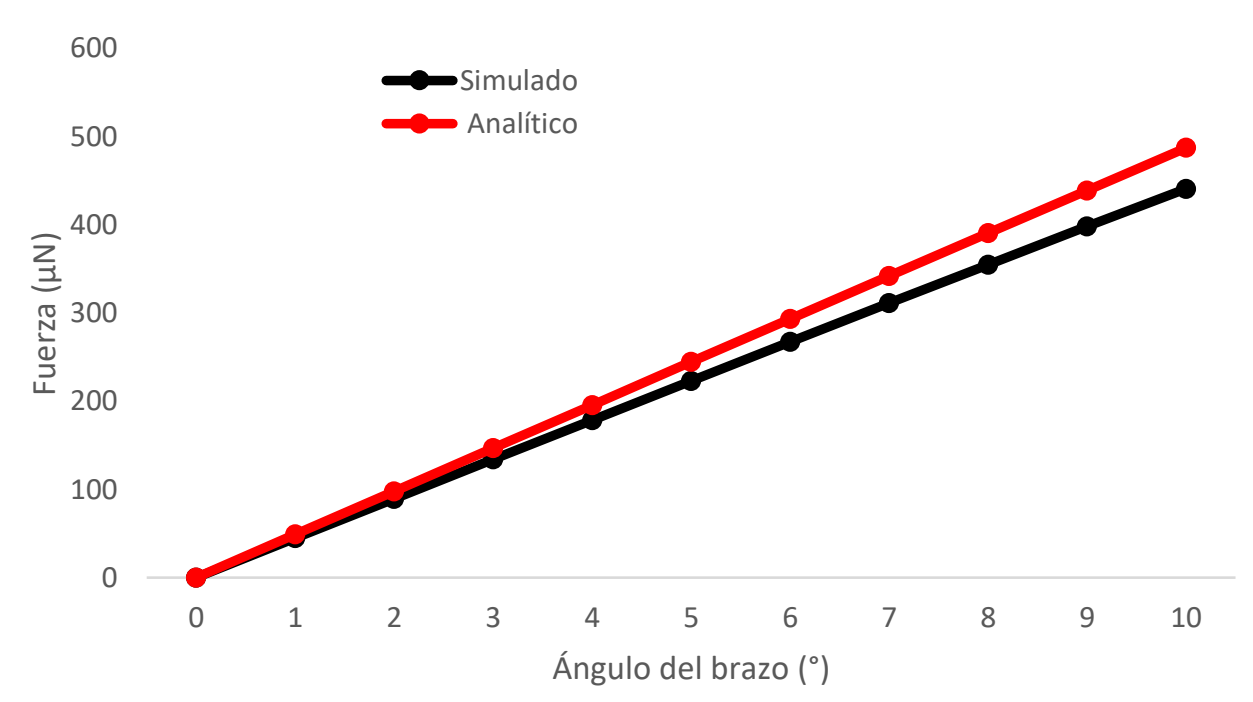

Fuente de Consulta: Elaboración propia

\subsection{Parametrización del grosor del actuador chevrón}

En este caso, se considera nuevamente a un chevrón de 2 brazos, con las características señaladas en la Tabla 7.2. En el gráfico 7.8, se muestran los valores de la fuerza al variar al grosor del dispositivo. Se observa una variación lineal creciente de $2 \mu \mathrm{m}$ a $30 \mu \mathrm{m}$. En este último valor, se tiene un error de $8.9 \%$. En la literatura se encuentra comúnmente a grosores menores a $5 \mu \mathrm{m}$, en la simulación de dispositivos. En nuestro caso, seleccionamos $15 \mu \mathrm{m}$.

De acuerdo con (Potekhina \& Wang, 2019), la rigidez del actuador está influenciada por el grosor, en concordancia con la ecuación 6. 
Gráfico 7.8 Grosor del actuador contra fuerza

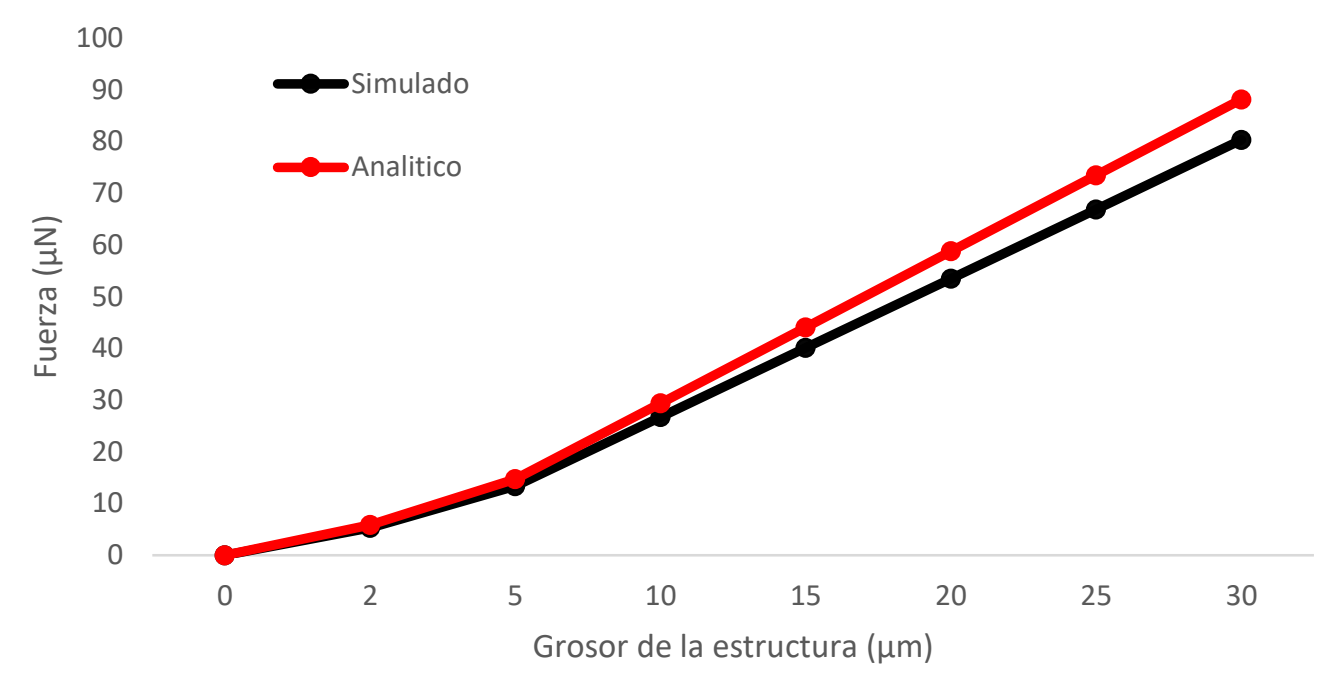

Fuente de Consulta: Elaboración propia

Con relación al grosor del dispositivo y sus efectos sobre el desplazamiento, de acuerdo con la ecuación 1, este efecto es muy pequeño, por lo que, no se incluye el gráfico. De manera indirecta, el grosor se incluye en el cálculo del momento de inercia. En la simulación, se obtiene una efecto similar.

\subsection{Parametrización del número de brazos del actuador chevrón}

El Gráfico 7.9 muestra la relación entre la fuerza y el número de brazos. Los datos mostrados para cada par de brazos, simulados y analítico, se obtuvieron por separado, de manera puntual. Aunque la fuerza se incrementa linealmente con el incremento en el número de brazos, suelen considerarse en general no más allá de 16. Arriba de este número puede generarse una flecha muy larga, lo que provocaría una degradación en el rendimiento, de acuerdo con (Potekhina \& Wang, 2019), pues se incrementaría la rigidez. En este caso, el mayor error obtenido fue de $9.39 \%$ para 16 brazos.

Gráfico 7.9 Número de brazos del actuador contra fuerza

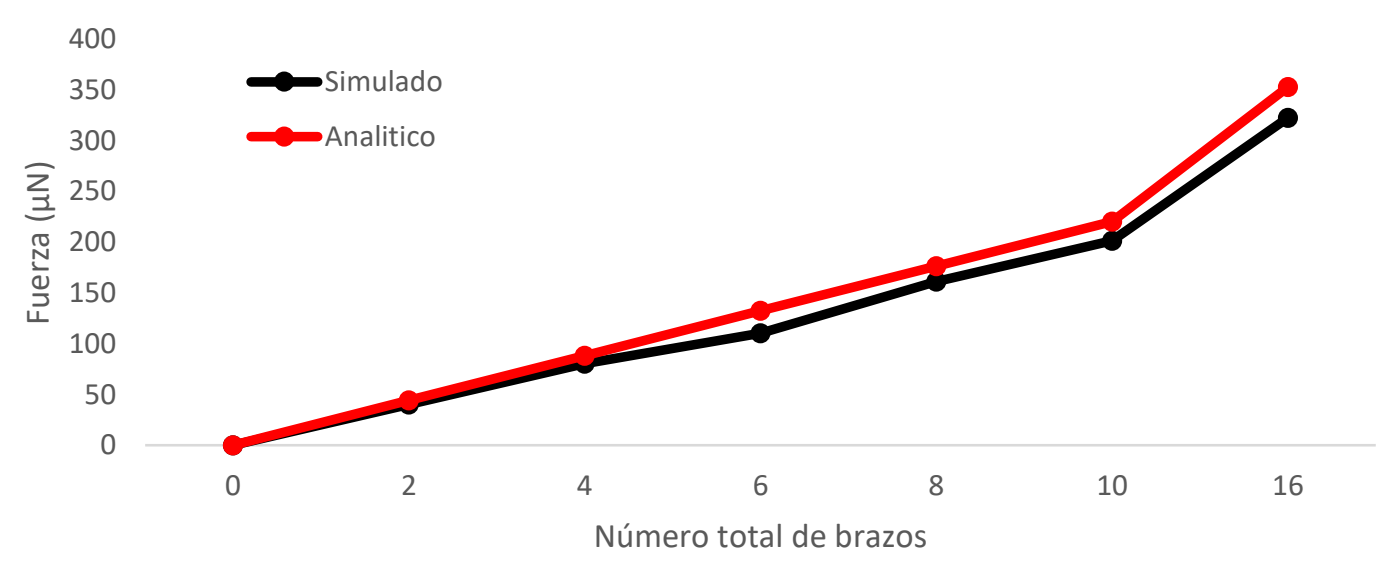

Fuente de Consulta: Elaboración propia

Con relación al número de bazos totales del actuador y el desplazamiento, no se observó un cambio significativo, de 2 a 16 brazos el cambio fue apenas de $0.01 \mu \mathrm{m}$ (desde $3.93 \mu \mathrm{m}$ hasta $3.94 \mu \mathrm{m}$ ), por lo que no se incluyó el gráfico correspondiente. 


\subsection{Parametrización del largo de la flecha}

En este caso, no se hallaron aproximaciones analíticas, por lo que solo se consideró a la simulación. Este caso fue analizado en (Báez Álvarez, 2011), para varias longitudes, de manera discreta. Se coincide en que, al incrementar la longitud, se incrementa al desplazamiento, aunque no se trata de una relación totalmente lineal. El autor menciona además que, con el incremento en las dimensiones de la flecha, se reduce su temperatura.

Gráfico 7.10 Longitud de la flecha contra desplazamiento

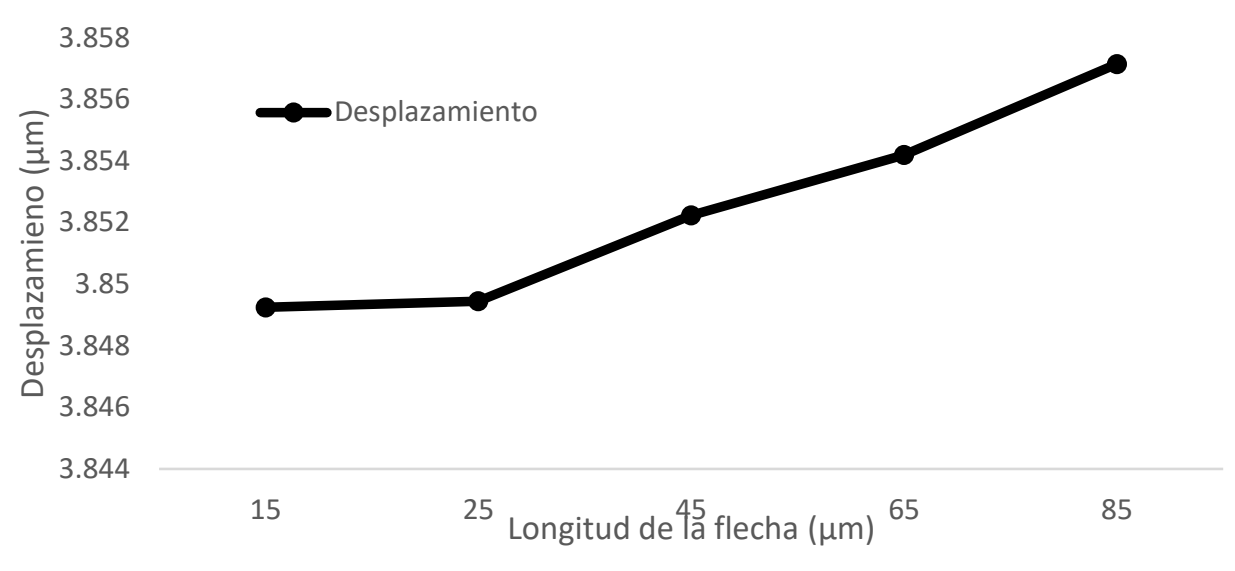

Fuente de Consulta: Elaboración propia

En los Gráficos 7.11 a 7.13 se consideró también, solamente a los resultados de la simulación.

$\mathrm{Al}$ incrementar la longitud de la flecha, se tiene un incremento considerable en la fuerza, entre 11 y $20 \mu \mathrm{m}$, y de ahí hasta $85 \mu \mathrm{m}$, no se observa un incremento notable. Para obtener un comportamiento estable, se sugiere utilizar largo de flecha de 25 a $90 \mu \mathrm{m}$. Nuevamente, la selección también depende fuertemente del número de brazos y su anchura. En (Báez Álvarez, 2011), pueden considerarse detalles sobre la distribución de la temperatura.

Gráfico 7.11 Longitud de la flecha contra fuerza

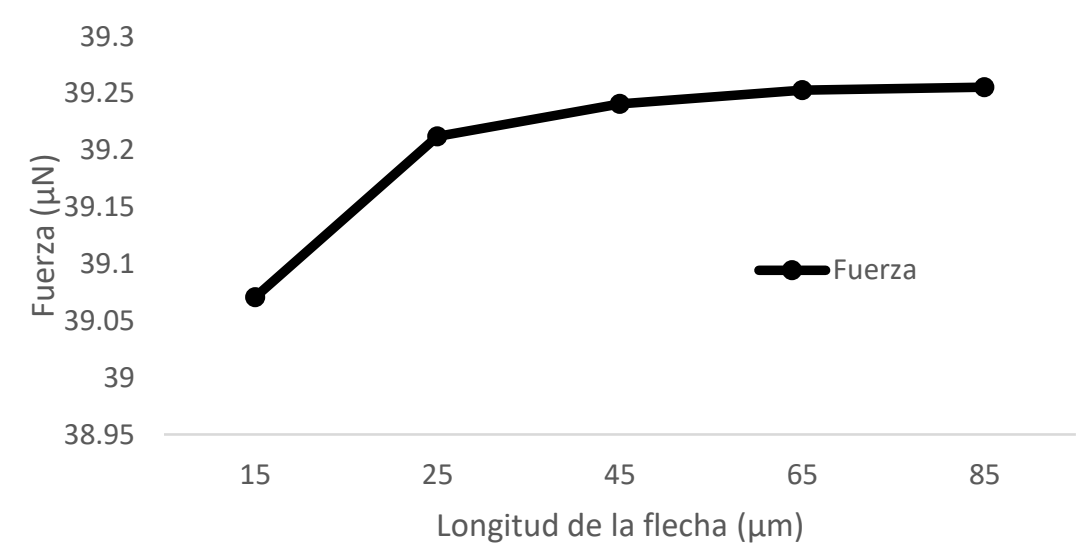

Fuente de Consulta: Elaboración propia

\subsection{Parametrización del ancho de la flecha}

Las variaciones del ancho de la flecha sobre el desplazamiento y la fuerza se muestran en los Gráficos 7.12 y 7.13. En ambos casos se aprecia la linealidad de la relación, con un comportamiento creciente. Sin embargo, los cambios en el rango de barrido no son significativos. 
Gráfico 7.12 Ancho de la flecha contra desplazamiento

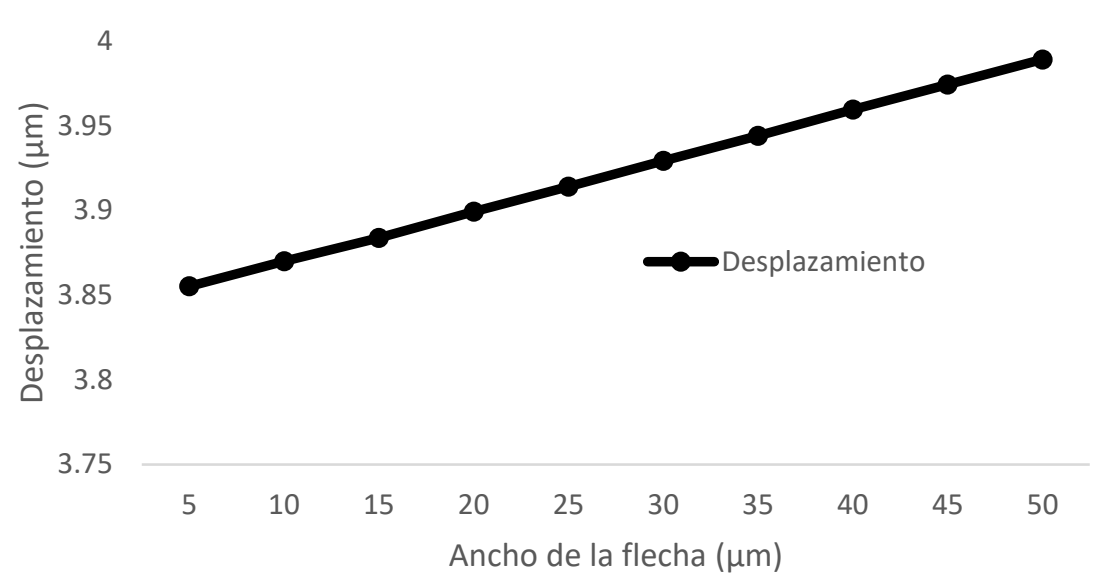

Fuente de Consulta: Elaboración propia

Gráfico 7.13 Ancho de la flecha contra fuerza

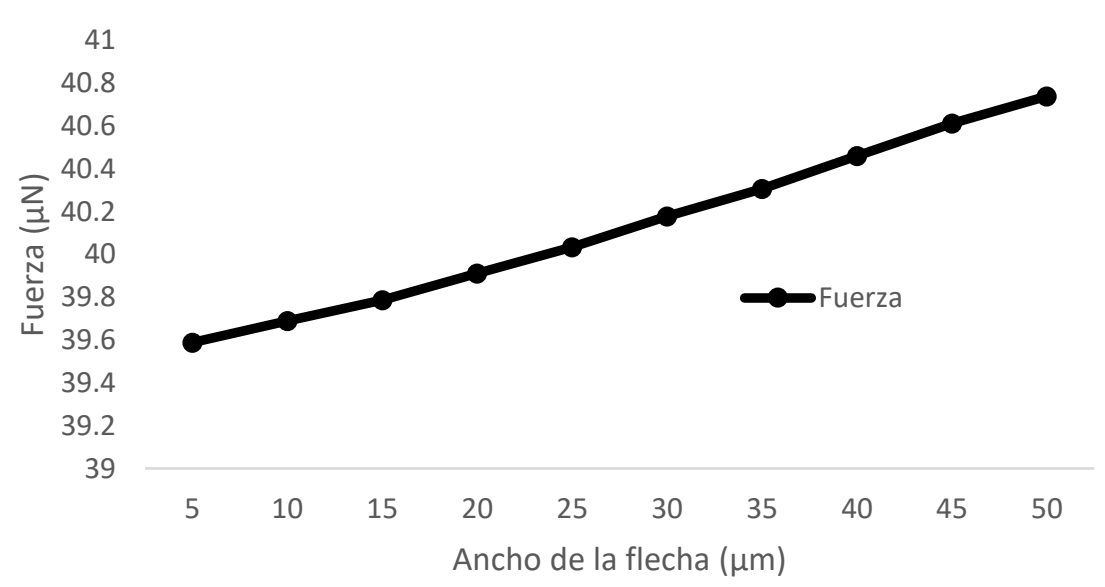

Fuente de Consulta: Elaboración propia

\subsection{Parametrización del gradiente de temperatura aplicado}

En este caso, el mayor valor del error entre los resultados analíticos, obtenidos de la ecuación 2, y los obtenidos mediante simulación es del 18\%. En el Gráfico 7.14, se observa un comportamiento creciente, conforme se incrementa la temperatura, en el rango considerado. El valor del coeficiente de expansión térmica se mantuvo constante, como comúnmente se lleva a cabo en la literatura. En (Steiner, Hortschitz, Stifter, \& Kepli, 2014), las mediciones del desplazamiento de un actuador chevrón con respecto al cambio de temperatura, muestra tambien un comportamiento prácticamente lineal. El rango de trabajo del actuador, también depende de la aplicación a la que se destine, siendo deseable emplear estrategias de reducción de temperatura, en el caso de objetivos de sujeción sensibles, cuando se utiliza al chevrón como medio de actuación de micropinzas. 
Gráfico 7.14 Gradiente de temperatura vs desplazamiento

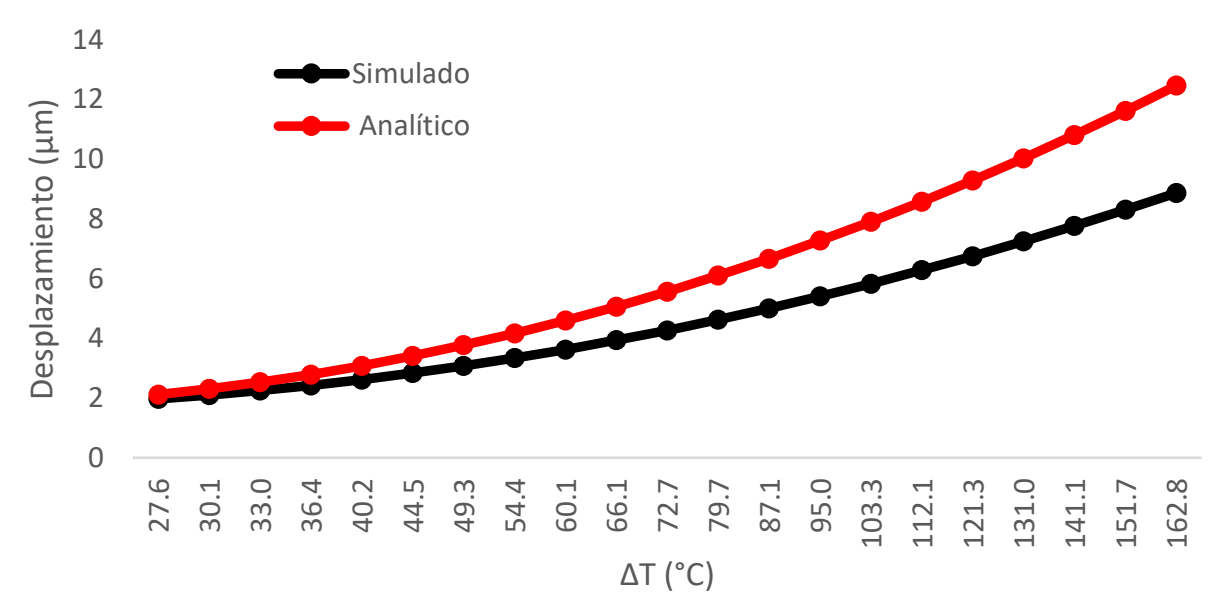

Fuente de Consulta: Elaboración propia

En el gráfico 7.15 se observa la relación de la temperatura con la fuerza, con un comportamiento creciente y lineal. El error mayor entre las aproximaciones analítica y mediante simulación corresponde a $41.5 \%$. Los incrementos observados en la simulación no son tan considerables, como en el caso analítico. Cabe señalar, que nuevamente, se sugiere trabajar en un rango de temperatura no elevado, de acuerdo con los elementos con los que se interaccione.

Gráfico 7.15 Gradiente de temperatura vs fuerza

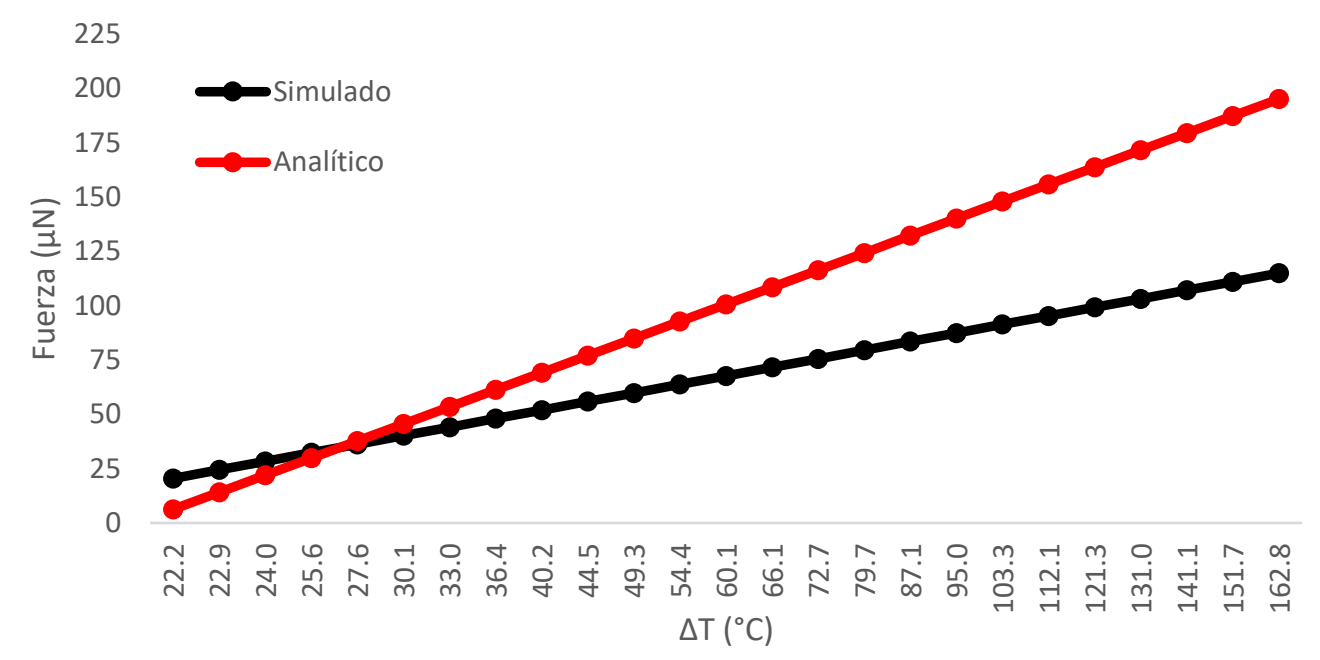

Fuente de Consulta: Elaboración propia

Cabe señalar que, con la finalidad de obtener un análisis más preciso, del efecto de la temperatura, es necesario considerar, en un trabajo futuro, que el coeficiente de expansión térmica no es constante con el cambio en la temperatura, por lo que, es deseable utilizar la expresión desarrollada en (Swenson, 1983), o bien, por los valores discretos que proporciona. Además, de realizar una búsqueda sobre este coeficiente, para temperaturas mayores a $100^{\circ} \mathrm{C}, \mathrm{o}$ expresiones alternativas.

La constante de rigidez no muestra afectaciones con la temperatura (Gráfico 7.16), con un error entre las aproximaciones analítica y los resultados de la simulación, tan solo de $0.09 \%$. 
Gráfico 7.16 Gradiente de temperatura vs rigidez

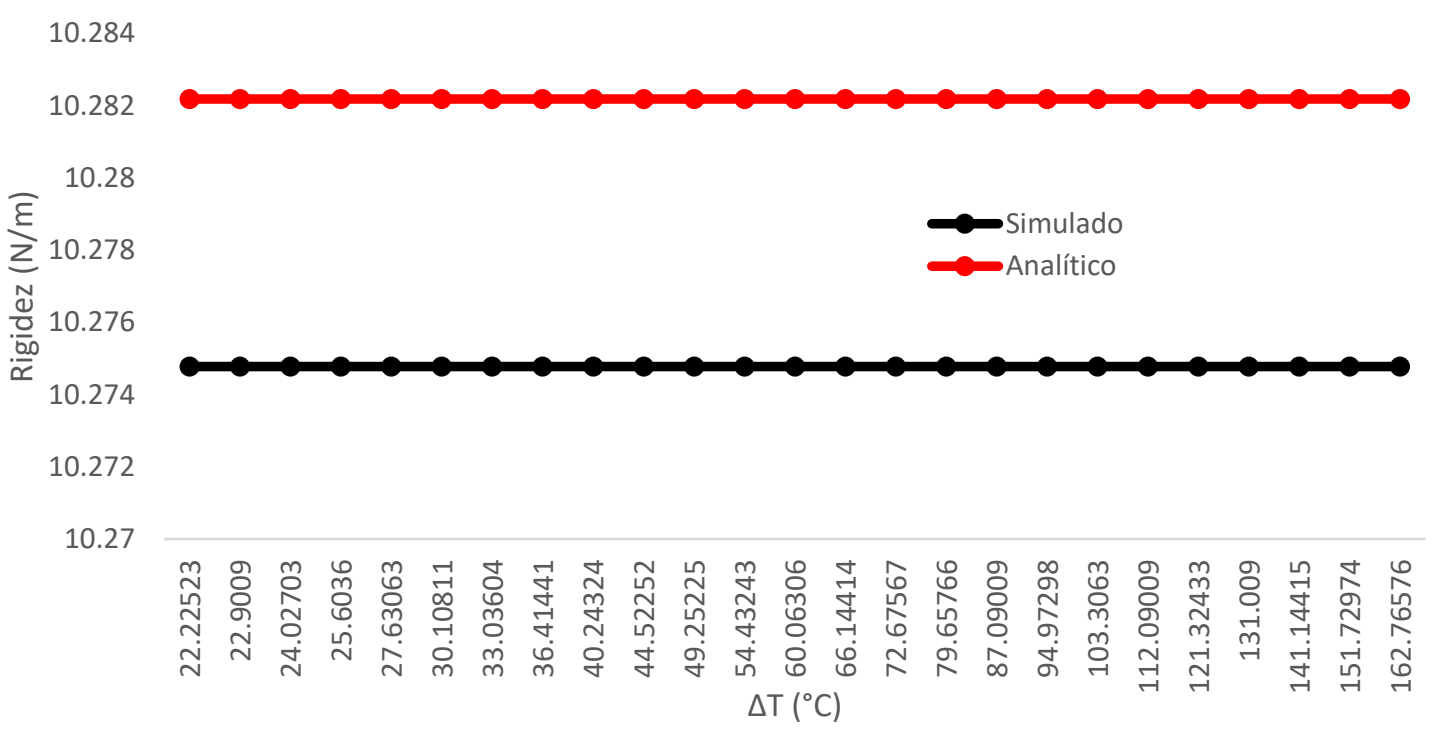

Fuente de Consulta: Elaboración propia

\subsection{Anexo}

Proceso de parametrización en Ansys Workbench del ancho del brazo, obteniendo el desplazamiento y la fuerza generados en la flecha del actuador chevrón

Se presenta este caso como ejemplo de parametrización. Los demás casos, se llevan a cabo de manera similar.

\section{Paso 1. Elaboración de la geometría en el área de trabajo de Project}

En esta etapa, se parametriza el ancho del brazo. Al abrirse la ventana Geometry (Figura 7.2), con clic derecho en el plano $\mathbf{X Y} \rightarrow$ sketch $\mathbf{1} \rightarrow$ details view (sección inferior derecha de la ventana) (Figura A.2), se selecciona la casilla V1, correspondiente al ancho del microactuador, y automáticamente el recuadro se rellena con la letra $\mathbf{D}$, al mismo tiempo, se despliega una ventana, donde se solicita colocar un nombre o identificación del parámetro a crear.

Figura 7.2 Secuencia de pasos para la parametrización geométrica

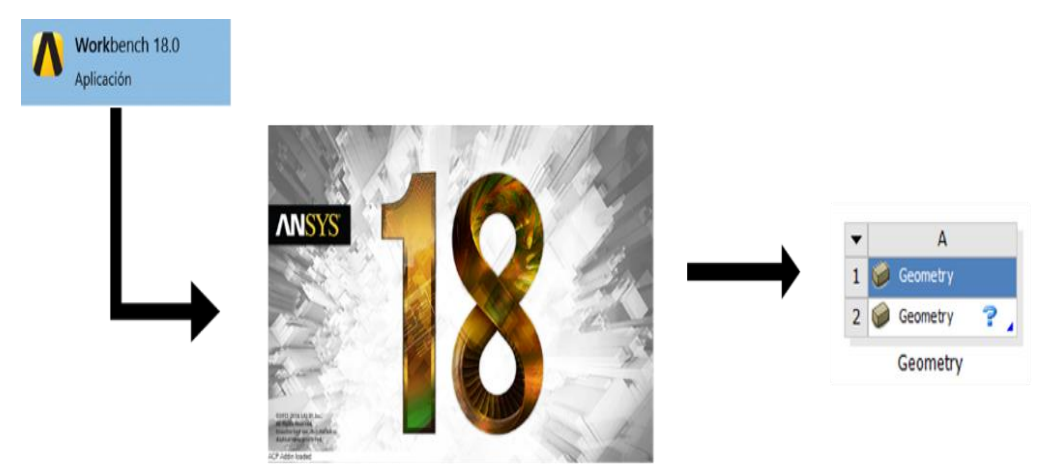




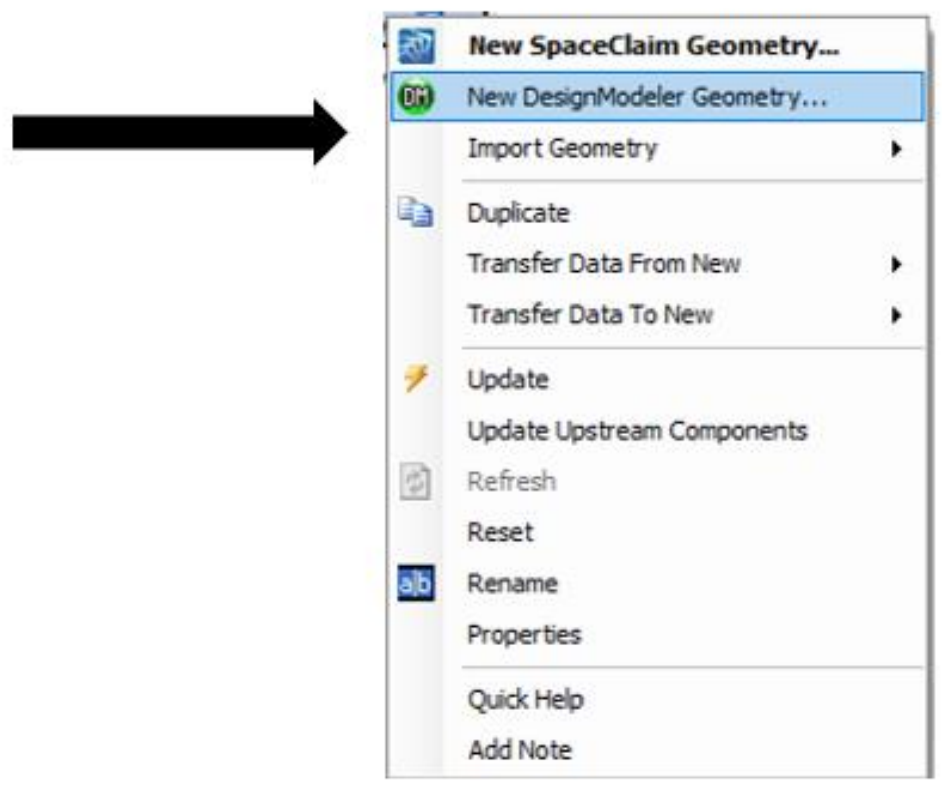

Fuente de Consulta: Elaboración propia

Figura 7.3 (a) Ventana de asignación para un identificador del parámetro. (b) Geometría del microactuador chevrón

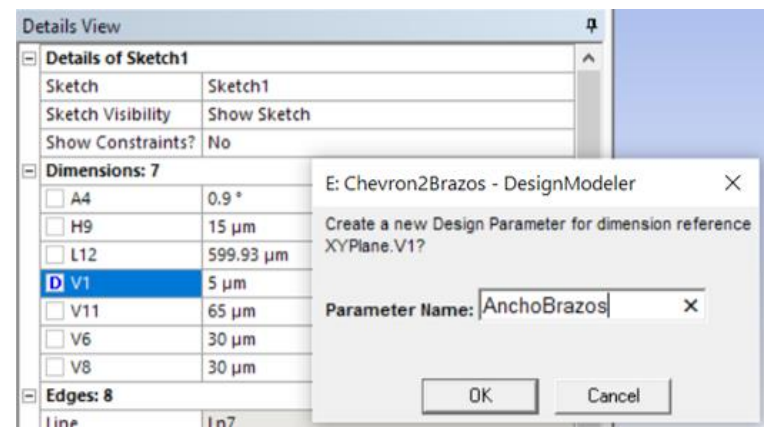

(a)

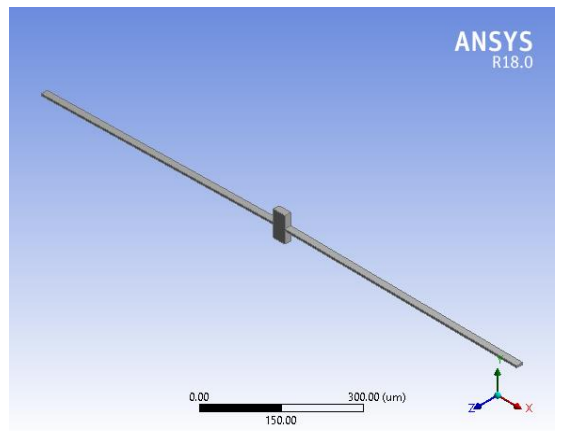

(b)

Fuente de Consulta: Elaboración propia

\section{Paso 2. Establecimiento de la variable a parametrizar, asignación de static structural para el cálculo de desplazamiento y fuerza}

Cerramos la ventana de la geometría y en Project Schematic damos doble clic sobre Geometry, mostrándose la ventana de la Figura 7.4. En la columna B se ubica el ancho de la geometría que estará variando, y que permitirá observar el comportamiento, de acuerdo con las variables de desempeño consideradas (fuerza y desplazamiento).

Figura 7.4 Tabla de diseño de puntos para la parametrización

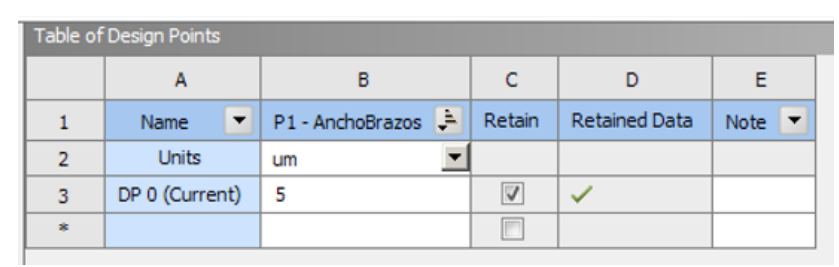

Fuente de Consulta: Elaboración propia

De Analysis Systems seleccionamos y arrastramos Steady-state Thermal y lo posicionamos a un costado de Geometry, doble clic en el Engineering Data y se asigna el material (Silicio). 
Posteriormente, con esa carga térmica y por las propias características del microactuador, éste desarrollará un desplazamiento proporcional a la fuerza. Arrastramos dos Static Structural que se unirán al Steady-State Thermal, que nos permite visualizar al desplazamiento y a la fuerza (Figura 7.5).

Figura 7.5 Árbol de herramientas para el análisis térmico del dispositivo microactuador

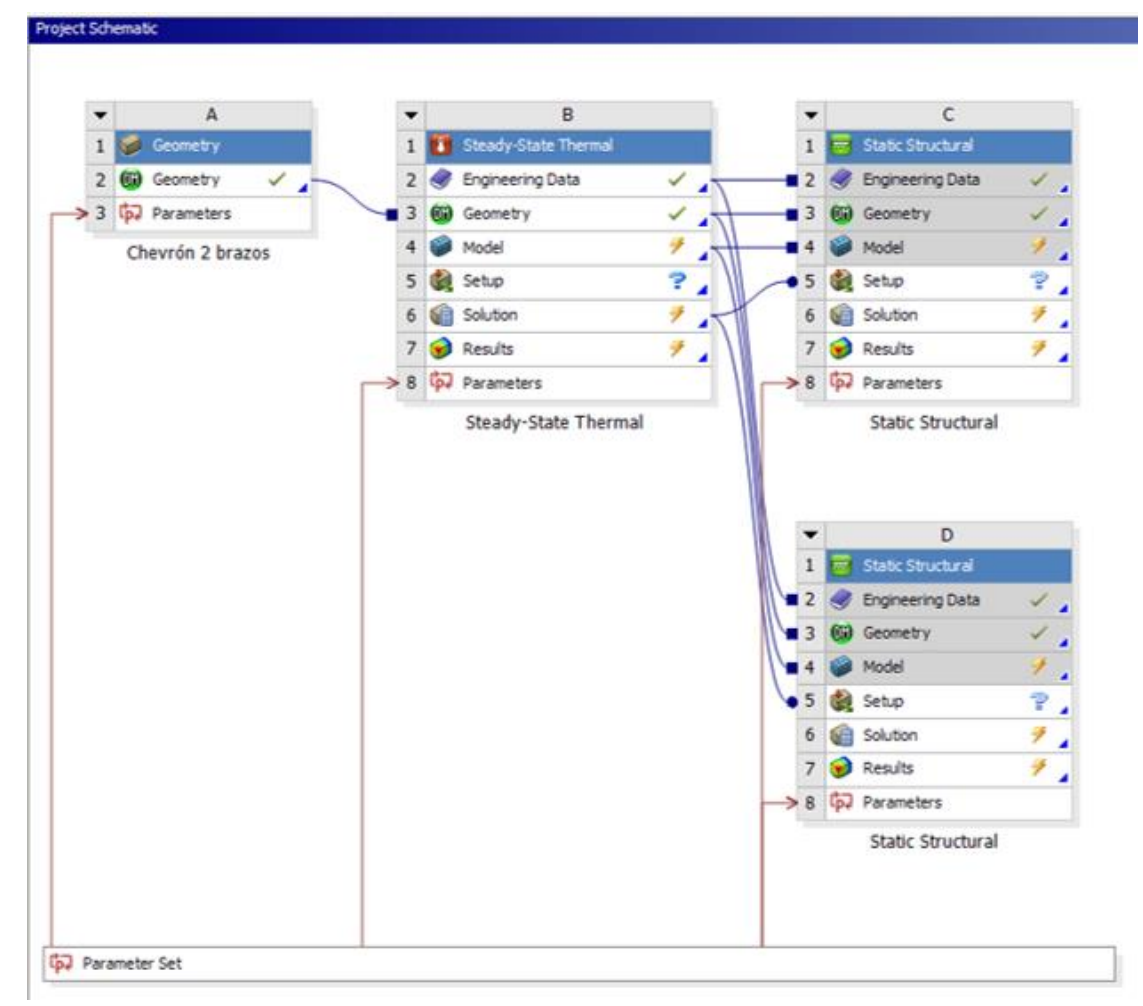

Fuente de Consulta: Elaboración propia

\section{Paso 3 Asignación del material, temperatura y mallado del dispositivo}

Al dar doble clic en Model del Steady-state Thermal, se visualiza la ventana del análisis termomecánico. Para obtener los resultados con las condiciones de frontera específicas, se debe llevar a cabo la secuencia de pasos mostrada en la Figura 7.6 
Figura 7.4 Árbol de análisis térmico. (a) Asignación del material. (b) Mallado. (c) En initial Temperature reajustar a $0^{\circ}$, renombramiento de Temperature 1 en Temperatura ambiente y asignación a $22{ }^{\circ} \mathrm{C}$. (d) Asignación de la parametrización de la carga térmica. (e) Oprimir Generate y visualización de la distribución de la temperatura

\begin{tabular}{|c|c|c|}
\hline Details of "Solid" & & \\
\hline Suppressed & No & A \\
\hline Stiffness Behavior & Flexible & \\
\hline Coordinate System & Default Coordinate System & \\
\hline Reference Temperature & By Environment & \\
\hline Behavior & None & \\
\hline \multicolumn{3}{|l|}{ Material } \\
\hline Assignment & Silicon_ok 4 & \\
\hline Nonlinear Effects & Yes & \\
\hline Thermal Strain Effects & Yes & \\
\hline \multicolumn{2}{|l|}{+ Bounding Box } & a \\
\hline
\end{tabular}

(a)

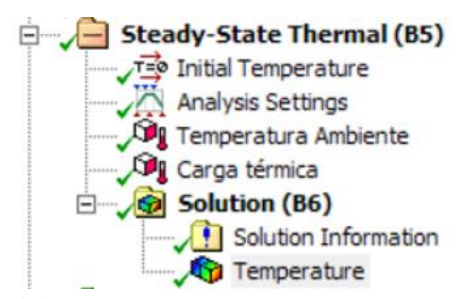

(c)
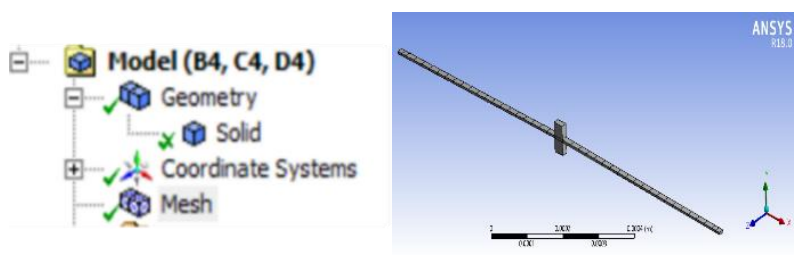

(b)

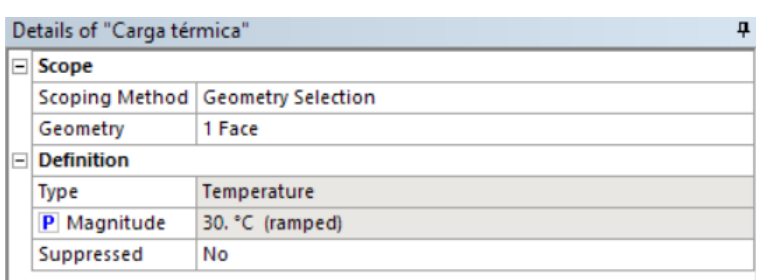

(d)

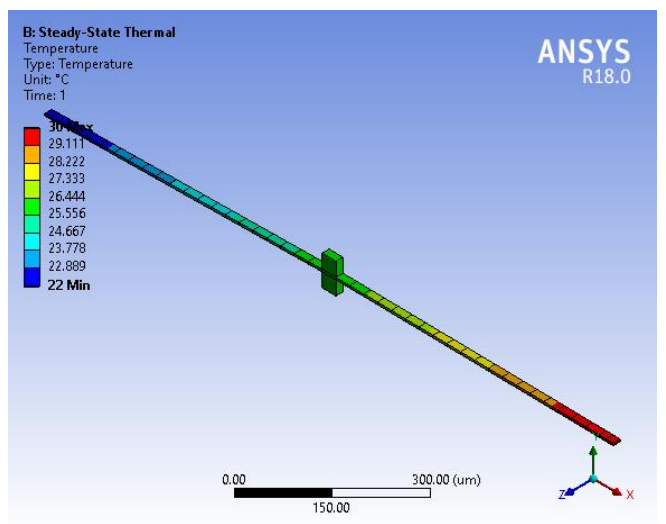

(e)

Fuente de Consulta: Elaboración propia

Puede observarse que, se eligió la malla por default, que debido a la geometría del dispositivo bajo análisis, es rectangular. En casos, en los que la geometría sea compleja y que implique la no convergencia de los elementos del mallado, se realiza un refinamiento de malla, que en general, es adaptativo.

Para un nivel macrométrico, en (Araujo, Briceño, \& Rondón, 2020), se analizan los casos de mallas triangulares y cuadradas, al utilizar el software ABAQUS, concluyendo que los resultados que obtienen en sus simulaciones son similares a los resultados experimentales. En (Cagua, Haro, \& Pilatasig, 2020), se realiza el análisis mediante Elemento Finito, para vigas de acople, y se llega a la conclusión de que la simulación es una herramienta adecuada para el análisis, pero que es necesaria la experimentación.

\section{El Paso 4 Obtención del desplazamiento y la fuerza de reacción}

El desplazamiento se obtiene a partir del árbol de análisis mostrado en la Figura 7.7 
Figura 7.7 Árbol de análisis estático estructural. (a) En static structural asignar $22{ }^{\circ} \mathrm{C}$, en la ventana de detalles, y clic derecho $\rightarrow$ Insert $\rightarrow$ Fixed Support, asignarlo en las anclas. (b) En Definition $\rightarrow$ Type, colocar Directional deformation y en orientation, elegir $Y$ axis. Se selecciona la parametrización del desplazamiento máximo. (c) Visualizar el desplazamiento a partir de la carga térmica asignada

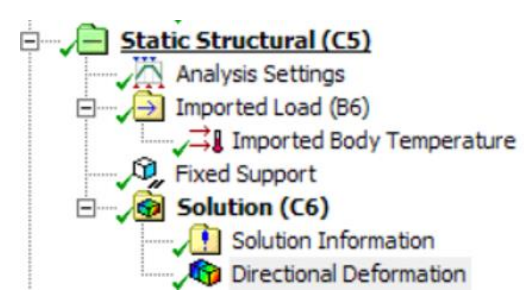

(a)

(c)

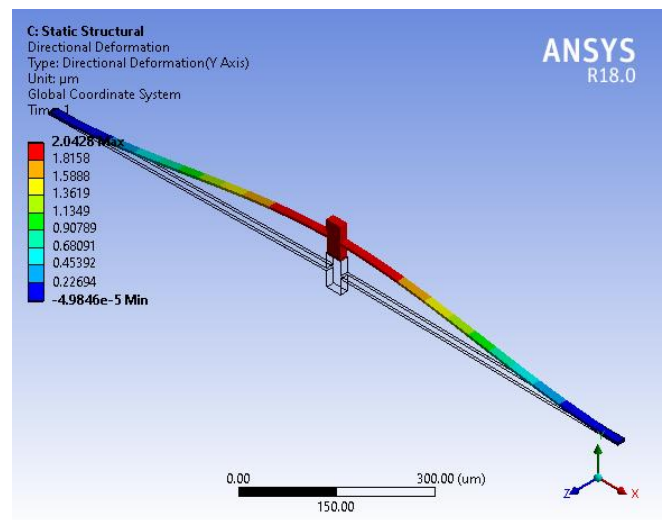

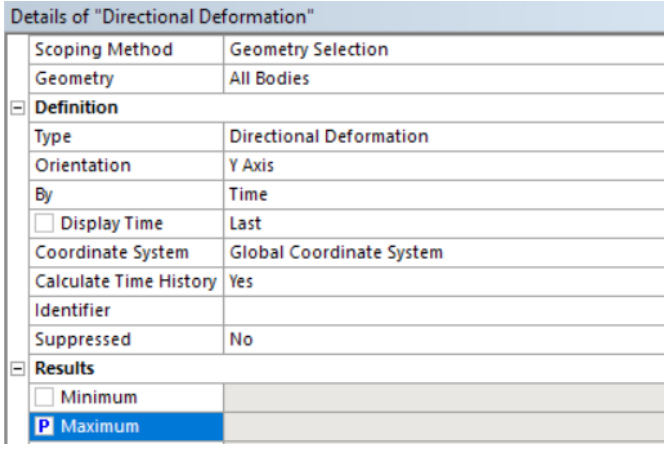

(b)

Fuente de Consulta: Elaboración propia

La fuerza de reacción se obtiene a partir del árbol de análisis mostrado en la Figura 7.8.

Figura 7.8 Árbol de análisis estático estructural para la obtención de la fuerza de reacción de la flecha.

(a) En static structural asignar $22{ }^{\circ} \mathrm{C}$, clic derecho y elegir fixed support dos veces, una es para las anclas y la otra que se debe asignar en la cara frontal de la flecha, clic derecho en solution $\rightarrow$ Insert $\rightarrow$ Probe $\rightarrow$ Force Reaction, en la ventana de detalles elegir Fixed support 2 y Generate. (b) En la sección de detalles en Results asignar la parametrización sobre el eje correspondiente. (c) Visualización de la fuerza de reacción.

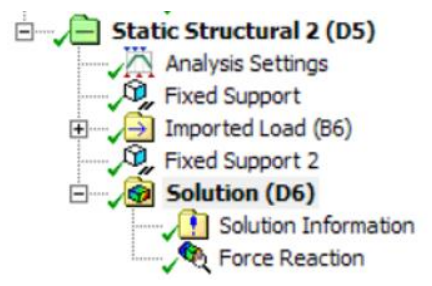

(a)

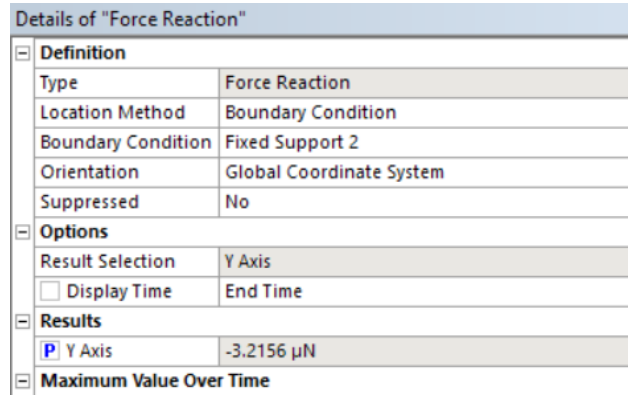

(b) 


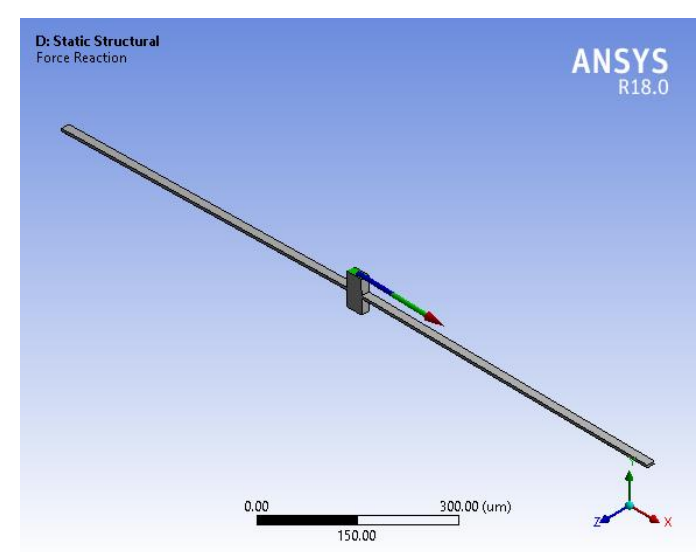

(c)

Fuente de Consulta: Elaboración propia.

\section{Paso 5. Despliegue numérico de los resultados}

Regresando a la ventana principal de Ansys Workbench en Project Schematic, dar doble clic en Parameter Set, parte inferior izquierda de la ventana, para realizar la asignación de la variación del ancho de los brazos del dispositivo manualmente, en un intervalo de $2 \mu \mathrm{m}$ a $11.5 \mu \mathrm{m}$, con la finalidad de tener un margen de seguridad. Se sigue el procedimiento mostrado en la Figura 7.9. Los resultados numéricos de la parametrización pueden exportarse a un graficador, que es lo que se hace en este capítulo, utilizando Origin.

Figura 7.9 (a) Árbol de las herramientas de análisis de project. (b) En la columna B se realiza la variación del parámetro, posteriormente en la parte superior izquierda en Update All Design Points, dar clic y se realizará la simulación paramétrica de las variables asignadas, realizándose la solución para desplazamiento y la fuerza

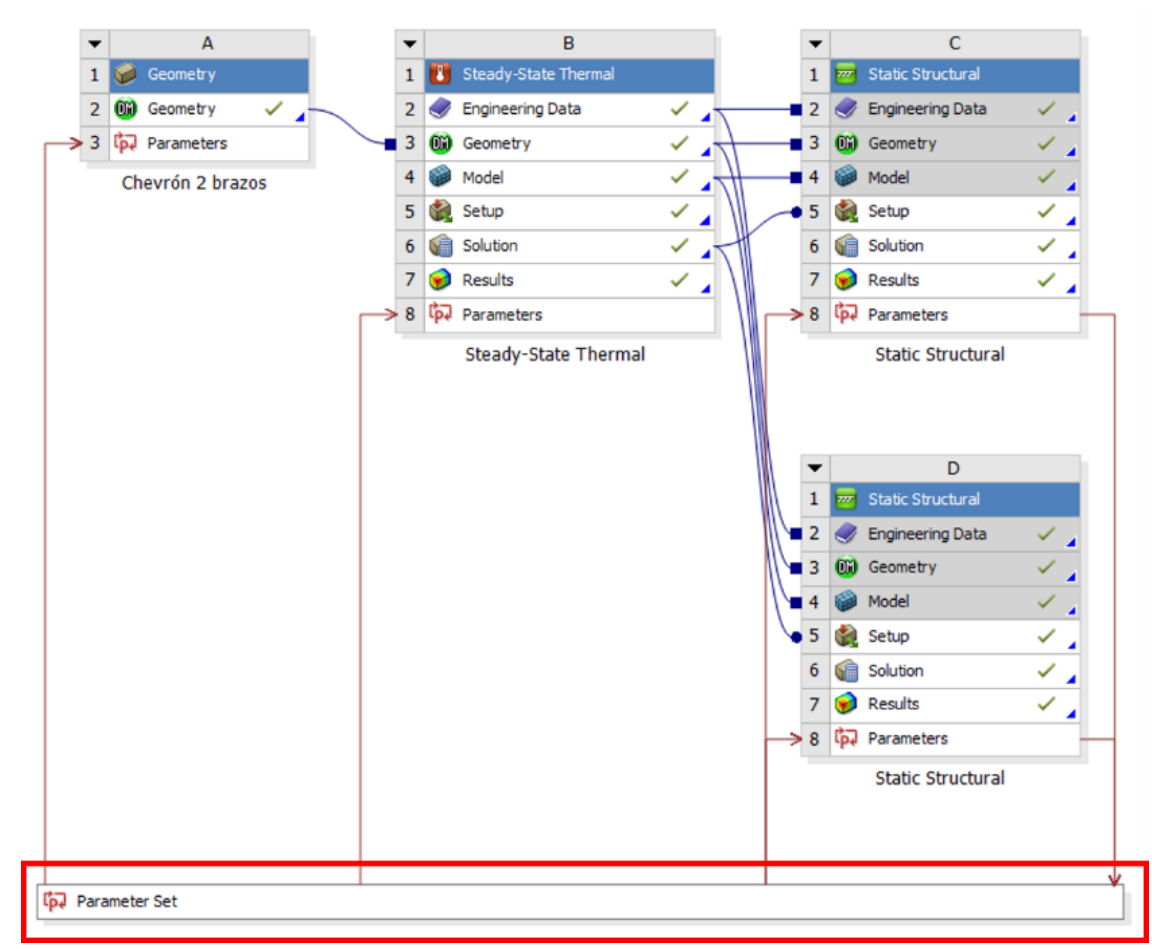

(a) 


\begin{tabular}{|c|c|c|c|c|c|c|c|}
\hline \multicolumn{7}{|c|}{ Table of Design Points } & \multirow{2}{*}{$=\frac{4}{D}$} \\
\hline & A & \multicolumn{2}{|l|}{ B } & \multicolumn{2}{|l|}{ c } & D & \\
\hline 1 & Name & P1 - ANCHO & 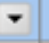 & P2 - Total Deformation Maximum & 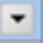 & \multicolumn{2}{|l|}{ P3 - Force Reaction Mir } \\
\hline 2 & Units & & $=$ & $\mathrm{m}$ & & \multicolumn{2}{|l|}{$\mathrm{N}$} \\
\hline 3 & DP 0 (Current) & 2 & & \multicolumn{2}{|l|}{$4.8233 E-06$} & \multicolumn{2}{|l|}{$-1.6126 E-05$} \\
\hline 4 & DP 1 & 2.5 & & \multicolumn{2}{|l|}{ 4.7094E-06 } & \multicolumn{2}{|l|}{$-2.014 \pi E-05$} \\
\hline 5 & DP 10 & 7 & & \multicolumn{2}{|l|}{$3.2443 E-06$} & \multicolumn{2}{|l|}{$-5.6125 E-05$} \\
\hline 6 & DP 11 & 7.5 & & \multicolumn{2}{|l|}{$3.0822 E-06$} & \multicolumn{2}{|l|}{$-6.0108 E-05$} \\
\hline 7 & DP 12 & 8 & & \multicolumn{2}{|l|}{ 2.926SE-06 } & \multicolumn{2}{|l|}{$-6.409 E-05$} \\
\hline 8 & DP 13 & 8.5 & & \multicolumn{2}{|l|}{$2.7769 E-06$} & \multicolumn{2}{|l|}{$-6.8098 E-05$} \\
\hline 9 & DP 14 & 9 & & \multicolumn{2}{|l|}{$2.636 E-06$} & \multicolumn{2}{|l|}{$-7.2191 E-05$} \\
\hline 10 & DP 15 & 9.5 & & \multicolumn{2}{|l|}{$2.5012 \mathrm{E}-06$} & \multicolumn{2}{|l|}{$-7.6173 E-05$} \\
\hline 11 & DP 16 & 10 & & \multicolumn{2}{|l|}{$2.3724 E-06$} & \multicolumn{2}{|l|}{$-8.017 E-05$} \\
\hline 12 & DP 17 & 10.5 & & $2.2508 E-06$ & & $-8.4142 E-05$ & \\
\hline 13 & DP 18 & 11 & & 2. $1365 E-06$ & & $-8.8152 E-05$ & \\
\hline 14 & DP 19 & 11.5 & & $2.0279 E-06$ & & $-9.2119 E-05$ & $\checkmark$ \\
\hline & & & & & & $>$ & \\
\hline Char & o data & & & & & $=4$ & $x$ \\
\hline
\end{tabular}

(b)

Fuente de Consulta: Elaboración propia

\subsection{Conclusiones}

En este capítulo, se analizó la parametrización de los elementos de un actuador chevrón y se analizaron sus efectos sobre el desplazamiento y la fuerza de reacción en la flecha. Se presentan porcentajes de error, entre los resultados analíticos y los obtenidos mediante la simulación, menores al 10\%, excepto en los casos de gradiente de temperatura contra fuerza y desplazamiento. Por lo que, es deseable que en un trabajo futuro se considere a la variación de los parámetros del material que dependen de la temperatura, en particular, del coeficiente de expansión térmica.

Con las parametrizaciones realizadas, pueden generarse recomendaciones que pueden ser útiles en el diseño de este tipo de actuadores. Las tendencias observadas de manera gráfica coinciden con los resultados que se hallaron en la literatura, correspondientes a otros grupos de trabajo, obtenidos mediante la simulación de manera discreta.

Los procesos de parametrización, al realizar barridos rápidos de manera automatizada, reducen considerablemente el tiempo para obtener información sobre la tendencia del desempeño que muestra cada uno de los elementos bajo análisis, previendo el comportamiento del dispositivo. En este caso, la parametrización de los elementos seleccionados y su efecto sobre el desplazamiento y la fuerza de reacción del actuador chevrón, permiten contar con criterios de selección de las dimensiones más adecuadas. Debe considerarse, además, que se satisfagan los requerimientos establecidos por la aplicación a la que se destinará el actuador, y también a los procesos de fabricación que se emplearán.

La simulación es una herramienta necesaria para obtener una aproximación sobre el desempeño de los dispositivos bajo análisis, para posteriormente implementarlos o fabricarlos, reduciendo el riesgo de posibles fallas que impliquen pérdidas económicas y de tiempo relacionadas. Una vez fabricados los prototipos, es posible realizar las pruebas experimentales para probar la factibilidad de su aplicación.

\subsection{Agradecimiento}

Esta investigación fue financiada por CONACyT, con número de referencia A1-S-33433, "Proyecto Apoyado por el Fondo Sectorial de Investigación para la Educación". 


\subsection{Referencias}

ANSYS Inc. (2011). Optimization in ANSYS Workbench. Obtenido de ANSYS Inc.: https://support.ansys.com/staticassets/ANSYS/Conference/Confidence/Houston/Downloads/optimizati on-in-ansys-workbench.pdf

ANSYS Inc. (s.f.). Parametric Analysis: The Key to Rapid, Robust Design. Obtenido de White paper: https://www.ansys.com/-/media/ansys/corporate/resourcelibrary/whitepaper/wp-parametricanalysis.pdf?la=en\&hash=4445A7D983C59F29A4E470B6D7721C2245948FF0.

Araujo, N., Briceño, Á., \& Rondón, A. (2020). Análisis comparativo de modelos bidimensionales con el método de elementos finitos y probetas fabricadas con mezclas asfálticas ensayadas con marco Lottman. Revista Ciencia e Ingeniería. Vol. 41, No. 1, 99-110.

Báez Álvarez, C. R. (2011). Caracterización de Microactuadores Tipo Chevrón Fabricados con la Tecnología PolyMEMS-INAOE. Tesis de Maestría. Tonantzintla, Puebla, Puebla, México: INAOE.

Báez Álvarez, C. R., Linares Aranda, M., Calleja Arriaga, W., \& Molina Reyes, J. (2013). Effect of Changing the Material and Device's Properties on the Performance of Polysilicon-Based MicroActuators. International Conference on Electrical Engineering, Computing Science and Automatic Control (págs. 415-420). CDMX: IEEE. DOI: 10.1109/ICEEE.2013.6676054.

Baracu, A., Voicu, R., Müller, R., Avram, A., Pustan, M., Radu, C., . . Dudescu, C. (2015). Design and fabrication of a MEMS chevron-type. INTERNATIONAL CONFERENCES AND EXHIBITION ON NANOTECHNOLOGIES AND ORGANIC ELECTRONICS (NANOTEXNOLOGY 2014): Proceedings of NN14 and ISFOE14, (págs. 25-30). Alemania.

Béjar, S. (2019). Análisis Paramétrico del Comportamiento a Fatiga de Pezas Torneadas eb Seco de la Aleación UNS A97075 (AL-ZN). MÁLAGA.

Cagua, B., Haro, A., \& Pilatasig, J. (2020). ANÁLISIS DEL COMPORTAMIENTO DE VIGAS DE ACOPLE CON DIFERENTE RELACIÓN DE LONGITUD VS PERALTE MEDIANTE ELEMENTOS FINITOS. Revista Internacional de Ingeniería de Estructuras Vol. 25, 2, 167-182, DOI:10.24133/riie.v25i2.1624.

Cauchi, M., Grech, I., Bertram, M., Pierluigi, M., Portelli , B., \& Sammut, N. (2019). Essential design and fabrication considerations for the reliable performance of an electrothermal MEMS microgripper. Microsystem Technologies, 16. DOI: 10.1007/s00542-019-04363-w.

Elbuken, C., Gui, L., Ren, C., Yavuz, M., \& Khamesee, M. (2008). Design and analysis of a polymeric photo-thermal microactuator. Sensors and Actuators A: Physical. Volume 147, Issue 1, 292-299. https://doi.org/10.1016/j.sna.2008.04.019.

González, O., Martínez, G., \& Graciano, C. (2020). Evaluación paramétrica de las principales variables geométricas en el diseño de un tren de aterrizaje para un avión no tripulado utilizando el método de los elementos finitos. Revista UIS Ingenierías Vol. 19, $n .{ }^{\circ} 2$, , 149-160.

Huber, J. E., Fleck, N. A., \& Ashby, M. F. (1997). The selection of mechanical actuators based on performance indices. Proc. of the Royal Society A. (págs. 2185-2205). London: The Royal Society Publishing. https://doi.org/10.1098/rspa.1997.0117.

Iamoni, S., \& Somà, A. (2014). Design of an electro-thermally actuated cell microgripper. Microsystem Technologies 20, 869-877. DOI: 10.1007/s00542-013-2065-8.

Judy, J. (2001). Microelectromechanical system (MEMS): Fabrication, design and applications. Smart Materials and Structures 10(6), 1115-1134. DOI: 10.1088/0964-1726/10/6/301. 
Jui-Chang, K., Hen-Wei, H., Shu-Wei, T., \& Yao-Joe, Y. (2014). A hydrogel-based intravascular microgripper manipulated using magnetic fields. Sensors and Actuators A: Physical 211, 121-130. https://doi.org/10.1016/j.sna.2014.02.028.

Kaajakari, V. (2009). Practical MEMS. Las Vegas, NV 89107: Small Gear Publishing.

Kwan, A., Song, S., Lu, X., Lu, L., Teh, Y.-K., Teh, Y.-F., . . Zeng, F. (2012). Improved design for an electrothermal in-plane microactuator. Microelectromech Syst Vol. 21 No. 3, 586-595.

Li, M., Zhou, Z., Yi, L., Wang, X., \& Adnan, S. (2019). Design of a test structure based on chevronshaped thermal actuator for in-situ measurement of the fracture strength of MEMS thin films. Science Direct, 163-168.

Potekhina, A., \& Wang, C. (2019). Review of Electrothermal Actuators and Applications. Actuators, MDPI Vol. 8(4), 28 páginas. https://doi.org/10.3390/act8040069.

Steiner, H., Hortschitz, W., Stifter, M., \& Kepli, F. (2014). Thermal actuators featuring large displacements for passive. Microsyst Technol. Vol. 20, 551-557.

Swenson, C. A. (1983). Recommended Values for the Thermal Expansivity of Silicon from 0 to $1000 \mathrm{~K}$. Journal of Physical and Chemical 12, 179, 179-182. https://doi.org/10.1063/1.555681.

Tazabekov, O. (2015). CMOS-MEMS RESONANT GATE Trasistor-Based Devices. University of Waterloo, Ontario, Canada.

Trung Hoang, K., Tien Nguyen, D., \& Hong, P. (2019). Impact of design parameters on working stability of the electrothermal V-shaped actuator. Microsystem Technologies 26, 1479-1487.

Vargas Chable, P., Ferrara Bello, C., Josue, S., Tecpoyotl Torres, M., \& Varona, J. (2019). A novel electrothermal compliance microgripper. 2019 International Conference on Mechatronics, Electronics and Automotive Engineering (ICMEAE) (págs. 74-78). Cuernavaca, Morelos: IEEE. DOI 10.1109/ICMEAE.2019.00021.

Vargas Chable, P., Ferrara Bello, C., Tecpoyotl Torres, M., Varona, J., \& Sandoval Reyes, J. (2019). A novel electrothermal compliance microgripper. IEEE, 6.

Vargas-Chable, P., Tecpoyotl-Torres, M., Robles-Casolco, S., \& Cabello-Ruiz, R. (2015). Structural optimization of an electrothermal chevron V- shape microactuator device. 2015 International Conference on Mechatronics, Electronics and Automotive Engineering (ICMEAE) (págs. 262-267). Cuernavaca, Morelos: IEEE. DOI: 10.1109/ICMA.2015.7237626.

Wang, Z., Shen., X., \& Chen, X. (2015). Design, modeling, and characterization of a MEMS electrothermal microgripper. Microsystem Technology 21, 2307-2314. DOI: 10.1007/s00542-014-24044.

Yang, S., \& Qingsong, X. (2017). A review on actuation and sensing techniques. Micro-Bio Robot 13, $1-14$.

Yole Developpement. (2019). STATUS OF THE MEMS INDUSTRY 2019. Market \& Technology. Yole Developpement, 3. Disponible en: https://yole-i-micronews-com.osu.eu-west2.outscale.com/uploads/2019/06/YD19031_Status_of_the_MEMS_industry_2019_Sample_Yole_Deve loppement.pdf.

Yong, Z., Corigliano, A., \& D Espinosa, H. (2006). A thermal actuator for nanoscale in situ microscopy testing: design and characterization. Journal of Micromechanics and Microengineering Volume 16, Number 2, 242-253.

Yukun, J., Nimping, J., \& Qingsong, X. (2014). A Dual-Axis Electrostatically Driven MEMS Microgripper. INTERNATIONAL JOURNAL OF ADVANCED ROBOTIC SYSTEM 11:187., 9 pages. https://doi.org/10.5772/59677. 
Zhu, Y., Corigliano, A., \& Espino, H. (2016). A thermal actuator for nanoscale in situ microscopy testing: design and characterization. J. Micromech. Microeng. 16, 242-253.

Zhuo, Z., Yueqing, Y., Xinyu, L., \& Xuping, Z. (2015). A Comparison Model of V- and Z-shaped Electrothermal Microactuators. 2015 IEEE International Conference on Mechatronics and Automation (ICMA) (pág. 6 pages. ). Beijing, China: IEEE. DOI: 10.1109/ICMA.2015.7237626. 